\title{
Electroactive polyamide/cotton fabrics for biomedical applications
}

\author{
Ana Raquel Bastos ${ }^{\mathrm{a}, \mathrm{b}, \mathrm{d}}$, Lucília Pereira da Silva ${ }^{\mathrm{a}, \mathrm{b}, \mathrm{c}}$, Vitor Pedro Gomes ${ }^{\mathrm{d}}$, Paulo E. Lopes ${ }^{\mathrm{e}}$, \\ Luísa Cidália Rodrigues $^{\mathrm{a}, \mathrm{b}}$, Rui Luís Reis ${ }^{\mathrm{a}, \mathrm{b}, \mathrm{c}}$, Vitor Manuel Correlo ${ }^{\mathrm{a}, \mathrm{b}, \mathrm{c}, *}$, António Pedro Souto ${ }^{\mathrm{d}}$ \\ a $3 B$ 's Research Group, I3Bs - Research Institute on Biomaterials, Biodegradables and Biomimetics, University of Minho, Headquarters of the European Institute of \\ Excellence on Tissue Engineering and Regenerative Medicine, AvePark, Parque de Ciência e Tecnologia, Zona Industrial da Gandra, $4805-017$ Barco, Guimarães, Portugal \\ b ICVS/3B's - PT Government Associated Laboratory, Portugal \\ ${ }^{\mathrm{c}}$ The Discoveries Centre for Regenerative and Precision Medicine, Headquarters at University of Minho, Avepark, 4805-017, Barco, Guimarães, Portugal \\ ${ }^{\mathrm{d}}$ University of Minho, Textile Engineering Department, Campus de Azurém, 4800-058, Guimarães, Portugal \\ ${ }^{\mathrm{e}}$ IPC - Institute for Polymers and Composites, Polymer Engineering Department, University of Minho, 4804-533, Guimarães, Portugal
}

\section{A R T I C L E I N F O}

\section{Keywords:}

Electroactive textiles

Polypyrrole

Polyaniline

Cotton

Polyamide

\begin{abstract}
A B S T R A C T
The latest advances on the development of wearables electrochemical sensors and biosensors has been revolutionizing healthcare, allowing a faster and specific diagnosis of pathological condition. The purpose of this work was to develop the first stage of wearable conductive based-textiles using natural (cotton) and synthetic (polyamide) fabrics composed of the conductive polypyrrole and polyaniline polymers.

Conductive polymers were polymerized in situ within fabrics using the correspondent monomers (pyrrole, Py and aniline, ANi) and an oxidizing agent (ammonium persulfate, APS). The obtained fabrics were characterized in terms of microstructure, hydrophobicity, chemical composition, color fastness of domestic and industrial washing, color fastness to rubbing and cytotoxicity. Optimal conductivity vales $\left(10^{-6}<\sigma<10^{-4}\right)$ were attained in PPy and PANi fabrics using 2:1 ratio (0.5 M Py and 0.25 M APS) and 1:1 ratio (0.5 M ANi and $0.5 \mathrm{M}$ APS), respectively. Textiles maintained their morphological integrity upon the polymerization process and, in some conditions, presented hydrophobicity $\left(\theta>90^{\circ}\right.$ for PA/CO fabrics containing PPy and CO fabrics containing PANi; $\theta<90^{\circ}$ for Bleached PA and PA fabrics containing PANi). The surface and volumetric conductivities of fabrics containing PPy or PANi were not affected after the color fastness to domestic and industrial washing and to rubbing testing's, except $\mathrm{CO}$ fabrics containing PANi. Cell viability was higher than $\approx 70 \%$ in both synthetic and natural fabrics containing PPy or PANi, with the exception of natural fabrics containing PANi that revealed a cell viability less than $\approx 50 \%$.

In conclusion, this study demonstrates the development and characterization of conductive based-textiles using synthetic and natural fabrics containing PPy and PANi with great potential to be used in future biomedical applications.
\end{abstract}

\section{Introduction}

Chronic diseases like Diabetes [1] and respiratory diseases (e.g. sleep apnoea, asthma, allergies, and heart disease) [2,3] demand a daily monitoring of biological factors, such as glucose levels, the measurement of heart rate, breathing volume, snoring, ambient ozone concentration, ambient temperature and relative humidity. This need has triggered the development of non-invasive wearables electrochemical sensors and biosensors to promptly follow the pathological condition [4].

Non-invasive wearables electrochemical sensors and biosensors are actually in use for real time personal health monitoring, namely on electrolytes and/or metabolites release from sweat, tears, saliva, urine, skin interstitial fluid [4,5] and also, on heart rate, wrist pulse, motion, blood pressure, intraocular pressure, body temperature [6,7]. Non-invasive wearable electrochemical sensors are commonly covered/made by a substrate, as silicon or gold, which present limitations in terms of the final structure and/or design, due to their stiffness and lack of comfort [8]. In fact, a crucial feature of a useful and functional e-textile is their affinity and stretchability when in contact with the skin [9]. This has potentiated the development of stretchable substrates to support the devices/sensors and promote a more intimate contact with the skin [10]. Fabrics/Textiles, in particular, represent a great class of polymeric substrates in which a biosensor can be integrated. E-textiles

\footnotetext{
* Corresponding author. , 3B's Research Group - Biomaterials, Biodegradables and Biomimetics, University of Minho, 4805-017, Barco, Guimarãess, Portugal.

E-mail address: vitorcorrelo@i3bs.uminho.pt (V.M. Correlo).
} 
are naturally stable promoting a suitable protection of the sensing area due to their robustness, mechanical strain, durability/maintenance and flexibility $[11,12]$. Synthetictextiles possess low cost to synthesis, easy processability and tunable and improved physiochemical properties [13]. Natural textiles present other enhanced properties, such as low density and cost, renewability, biodegradability, non-cytotoxicity, good sorption properties, softness and affinity to skin [14,15]. Generally, natural fibers have a hydrophilic nature, while the synthetic ones are more hydrophobic, which directly influences the dimensional stability of the fabrics. For instance, if a biosensor/electronic is applied within the textile, a hydrophobic fabric decreases the probability of short circuits caused by sweating or high humidity. Whilst, if the biosensor/ electronic is attached to the surface, a hydrophilic nature avoids the buildup of static electricity [16]. Nevertheless, despite of the adequate flexibility and good mechanical stability, common textiles have a nonconductive nature. To overcome this limitation, conductive polymers have been combined with textiles. Polypyrrole (PPy) and Polyaniline (PANi) conductive polymers have been proposed for biomedical applications due to their rich redox chemistry/electroactivity, biocompatibility and environmental stability [17]. Zhao and co-workers developed a flexible sensor for monitoring respiration using a knitted cotton fabric containing an in situ polymerization of polypyrrole and polyurethane coating, showing the possibility to accurately measure the respiration frequency [18]. In a different study, researchers produced a strain sensor through the incorporation PANi, graphene nanoplatelets and a handful of silicon rubber onto elastic lycra fabric using the spincoating method. The developed sensor was able to detect and monitor the bending angle of a human finger [19].

In this sense, the purpose of this work was to develop the first stage of a wearable conductive based-textile using synthetic (polyamide) or natural (cotton) fabrics composed of Polypyrrole (PPy) and Polyaniline (PANi). Having this in mind, the polymerization method of the corresponding monomers pyrrole (Py) and aniline (ANi) to PPy and PANi was optimized by varying the monomer concentration, and the amount and time of the oxidizing agent (ammonium persulfate, APS). A polyether polyurethane commercial product was applied to form a continuous film, adherent to the substrate, to ensure/retain the conductive properties of the developed fabrics. Fabrics were then characterized in terms of macro and microstructure, hydrophilicity/hydrophobicity, chemical composition, color fastness to domestic and industrial washing and to rubbing and cytotoxicity.

\section{Experimental section}

\subsection{Materials}

Commercial bleached polyamide 6.6 and cotton were used as received, except polyamide which was posteriorly treated with plasma. Polyamide (PA) is a plain fabric presenting an area weight of $113 \mathrm{~g} / \mathrm{m}^{2}$; the warp is composed of 46 threads/cm with 8,5 tex while, the weft is composed of 32 threads/cm with 20 tex. Cotton (CO) is a plain fabric presenting an area weight of $113 \mathrm{~g} / \mathrm{m}^{2}$; the warp is composed of 31 threads/cm with 20 tex while, the weft is composed of 27 threads/cm with 20 tex. Pyrrole (Py, 98\%) monomer, aniline (ANi, $\geq 99.5 \%$ ) monomer and ammonium persulfate (APS) were purchased from Sigma (USA). Py and ANi were distilled before use. The BAYPRET ${ }^{\circledR}$ NANO-PU finishing product was purchased from Tanatex Chemicals (USA) and used as received.

\subsection{Dyeing of synthetic and natural fabrics upon monomers polymerization}

Fabrics dyeing protocol was optimized from a previous work developed at 3B's Research Group [20,21]. Synthetic or natural fabrics were immersed in a solution containing the monomers Py $(0.50,1.00$ or $1.50 \mathrm{M}$ in ultra-pure water) or ANi $(0.50,1.00$ or $1.50 \mathrm{M}$ in $1.00 \mathrm{M} \mathrm{HCl})$ for $30 \mathrm{~min}$ or $60 \mathrm{~min}$, at stirring conditions using an orbital shaker (KS
Table 1

Experimental conditions used to polymerize synthetic and natural fabrics using the monomers, Py or ANi, and the oxidizing agent, APS.

\begin{tabular}{lllll}
$\Delta \mathrm{t}$ Monomer (min) & 30 & & 60 & \\
$\Delta \mathrm{t}$ Monomer and APS (hr) & 3 & & 6 & \\
Oxidizing Agent, APS (M) & 0.25 & & 0.50 & \\
Monomers, Py or ANi (M) & 0.50 & 1.00 & & 1.50 \\
\hline
\end{tabular}

260 control, IKA) at $200 \mathrm{rpm}$. In a further step, fabrics were oxidized through the addition of the oxidizing agent APS at different concentrations $(0.25$ or $0.50 \mathrm{M})$ for different periods ( $3 \mathrm{hr}$ or $6 \mathrm{hr}$ ) to complete polymerization (Table 1). Following, fabrics were rinsed in running water and left to dry at room temperature (RT). Fabrics that were used in cell culture were sterilized before use by ethylene oxide.

\subsection{Plasma treatment of synthetic fabric}

Plasma treatment was applied using a semi-industrial machine (Softal/University of Minho) following a protocol developed by António Pedro Souto and co-workers [22,23]. As the amount of plasma dosage applied to the substrate is influenced by the power of discharge, velocity, and number of passages of the fabric between electrodes, the amount of plasma dosage was calculated using the following equation:

Dosage $=\frac{\mathrm{PxN}}{\mathrm{Vxw}}$

Where $\mathrm{P}=$ power (Watt); $\mathrm{N}=$ number of passages; $\mathrm{v}=$ velocity $\left(\mathrm{m} . \mathrm{min}^{-1}\right)$, and $\mathrm{w}=$ width of treatment $(0.5 \mathrm{~m})$.

Plasma dosage applied to the substrate was $33.3 \mathrm{~J} . \mathrm{m}^{-2}$, wherein the number of passages was 5 (in each side), the velocity was $5 \mathrm{~m} \cdot \mathrm{min}^{-1}$ and power discharge was $1000 \mathrm{~W}$.

\subsection{Finishing process of synthetic and natural fabrics}

Fabrics containing PPy or PANi were finished using a padder machine containing a solution of BAYPRET $^{\circledR}$ NANO-PU (Tanatex Chemicals, EUA) in a concentration of 80 g.L $\mathrm{L}^{-1}$. The pressure used was 3atm, the velocity was $1.50 \mathrm{~m} \mathrm{~min}^{-1}$ and fabrics were passed through a foulard 3 times. Fabrics were weight in the dried (Wd) and wet (Ww) state in order to calculate the absorption rate of the dyeing.

Absorption Rate $(\%)=\frac{\mathrm{Ww}-\mathrm{Wd}}{\mathrm{Wd}} \times 100$

Next, fabrics were placed in a drying oven at $130^{\circ}$ for $5 \mathrm{~min}$.

\subsection{Electrical conductivity measurements}

The electrical conductivity of the synthetic and natural fabrics was measured using a Picoammeter 6487 with 8009 electrodes (Keithley, USA) at RT. The equipment determined the resistance, $R[\Omega]$ through Ohm's law, according to the following equation:

$\mathbf{V}=\mathrm{R} \mathrm{xI}$

Accordingly, $\mathrm{V}$ corresponds to the applied voltage $[\mathrm{V}]$ and I to the current across the fabrics [A]. In the present case, the area was $53.40 \mathrm{~cm}^{2}$ and the thickness (l) $[\mathrm{cm}]$ of each fabric was measured using a digital caliper (Mitutoyo, Japan). Surface and volumetric conductivities were measured using a voltage of $1 \mathrm{~V}$ in a range of 100 measurements.

Surface resistivity: $\rho=53.4 \times \frac{\mathrm{V}}{\mathrm{I}}$

Volume resistivity: $\rho=\frac{22.9}{1} \times \frac{\mathrm{V}}{\mathrm{I}}$

Electrical conductivity $(\boldsymbol{\sigma})$ was then calculated as the inverse of the resistivity $(\rho)$ : 
$\rho=\frac{1}{\rho}$

\subsection{Morphological analysis by scanning electron microscopy (SEM)}

Scanning electron microscopy (SEM, JSM-6010 LV, JEOL, Japan) was used to analyze the morphology of synthetic and natural fabrics bleached or dyed after monomers (Py or ANi) polymerization, and after application of the finishing product. Samples were cut in small pieces and sputtered coated with platinum ( $3 \mathrm{~nm}$ of thickness) using a Sputter Coater Equipment (Model EM ACE600) from Leica; the acceleration voltage used was $10 \mathrm{kV}$.

\subsection{Contact angle measurements}

The contact angles of the water drops in synthetic and natural fabrics bleached and dyed with PPy or PANi was measured using Dataphysics equipment using OCA 15PLUS software with video system for the capturing of images in static mode. Manual mode was used, the water drop had a volume of $3000 \mu \mathrm{L}$, and the velocity was set at $5 \mu \mathrm{Ls}^{-1}$.

\subsection{X-ray photoelectron spectroscopy (XPS analysis)}

The chemical composition of the synthetic and natural fabrics bleached and dyed upon monomers (Py or ANi) polymerization was examined by X-ray photoelectron spectroscopy (XPS) surface measurements. The C1s, O1s, S2p, N1s and survey spectra were recorded using a Kratos Axis-Supra instrument. The monochromatic X-ray source $\mathrm{Al} \mathrm{K \alpha}$ used was $1486.6 \mathrm{eV}$. The residual vacuum in the X-ray analysis chamber was maintained at $8.5 \times 10^{-9}$ torr. Fabrics were fixed to the sample holder with double sided carbon tape. Charge referencing was done by setting the binding energy of $\mathrm{C} 1 \mathrm{~s}$ photo peak at $285.0 \mathrm{eV} \mathrm{C} 1 \mathrm{~s}$ hydrocarbon peak. Charge compensation was employed to minimize surface changing to an electron flood gun. A wide scan survey spectrum was used to identify and quantify the elements in each fabric. High resolution narrow scans were used to build the chemical state assessment. Data analysis and atomic quantification were determined from the XPS peak areas using the ESCApe software supplied by the manufacturer Kratos Analytical.

\subsection{Color fastness testing in textiles - NP EN ISO 105}

\subsubsection{Color fastness of domestic and industrial washing - NP EN ISO 105:C06:1994}

Specimens and multifiber fabric were cut with $100 \mathrm{~mm}$ of height and $40 \mathrm{~mm}$ of length. The test used was C2S using $1 \mathrm{~g} . \mathrm{L}^{-1}$ of sodium perborate at $60^{\circ}$ in a final volume of $50 \mathrm{~mL}$ containing 25 steel balls at $\mathrm{pH}$ of $10,50 \pm 0,10$ for $30 \mathrm{~min}$ in a Washtec-P machine. The solution was prepared using ECE standard/reference detergent without optical brightener ( 4 g.L $\mathrm{L}^{-1}$ using $3^{\circ}$ water) with sodium perborate. Next, testpieces were left to dry at RT. Staining of adjacent fabrics that occurred with washing of a specimen were measured using a Spectraflash 600 (Datacolor) diffuse reflectance spectrophotometer at standard illuminant D65 (LAV/Spec. Incl., d/8, D65/10 ${ }^{\circ}$ ) according to ISO Standard.

\subsubsection{Color fastness to rubbing - NP EN ISO 105:X12:2003}

Specimens of textiles were cut with $180 \mathrm{~mm}$ of height and $60 \mathrm{~mm}$ of length. Cotton was used like a rubbing fabric to be in contact with each specimen. The color fastness to rubbing testing was performed in dry conditions using a crockmeter with 10 rotary movements. After rubbing, the staining of cotton adjacent fabric was evaluated/determined using a Spectraflash 600 (Datacolor) diffuse reflectance spectrophotometer at standard illuminant D65 (LAV/Spec. Incl., d/8, D65/10 ) according to ISO Standard.
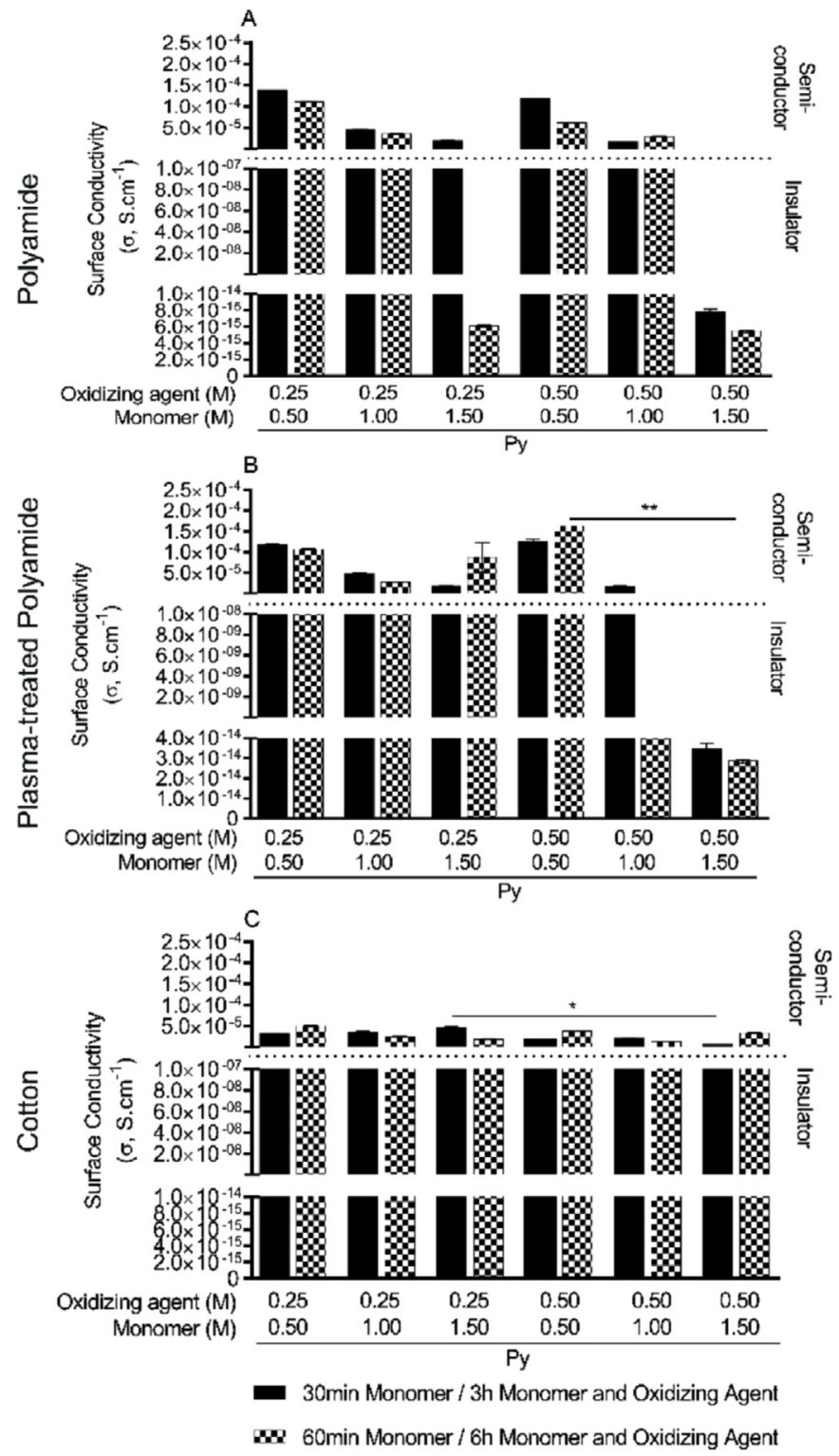

Fig. 1. Surface conductivity of polyamide (A), plasma-treated polyamide (B) and cotton $(\mathrm{C})$ fabrics dyed upon pyrrole polymerization. Different concentrations of oxidizing agent (APS, 0.25 and $0.50 \mathrm{M}$ ) and monomer (Py, 0.50, 1.00 and $1.50 \mathrm{M}$ ), as well as different periods of polymerization $(30 \mathrm{~min} / 60 \mathrm{~min}$ in Py followed by $3 \mathrm{hr} / 6 \mathrm{hr}$ in Py and APS) were used. Statistical analysis was performed using a Kruskal-Wallis test followed by Dunn's test. Polymerization time and different monomer/oxidizing agent concentrations were compared between them and analyzed.

\subsection{Cytotoxicity assay}

The cytotoxic effect of potential leachables from fabrics containing PPy or PANi were evaluated according to the ISO 10993-5:2009 (Biological evaluation of medical devices - Part 5: Tests for in vitro cytotoxicity) using a L929 mouse fibroblasts line (L929, European Collection of Cell Cultures). Samples preparation was done in accordance with ISO 10993-12; as fabrics thickness is less than $0.50 \mathrm{~mm}$, the extraction ratio (surface area) used was $6 \mathrm{~cm}^{2} / \mathrm{mL}$. Additionally, total extracts $(100 \%)$ and diluted extracts $(70 \%, 50 \%$ and $30 \%)$ were prepared by immersion of the fabrics in Dulbecco's modified eagle's medium - low glucose (DMEM, Sigma, USA) supplemented with $10 \%$ fetal bovine serum (FBS, Alfagene, Portugal), 1\% antibiotic/antimycotic (Alfagene, Portugal) at $37^{\circ}$ and stirring conditions for $24 \mathrm{hr}$. 

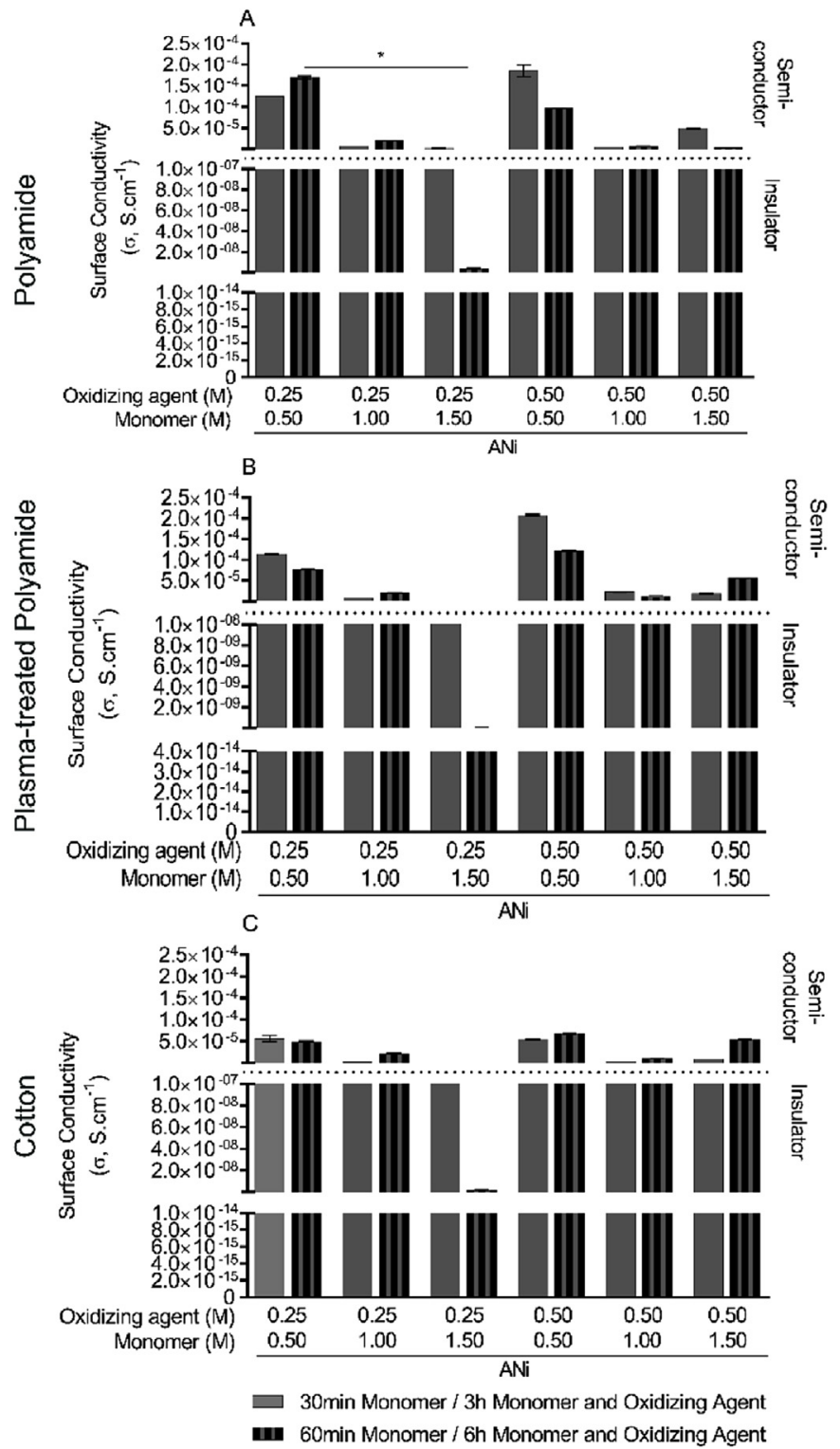

Fig. 2. Surface conductivity of polyamide (A), plasma-treated polyamide (B) and cotton $(C)$ fabrics dyed upon aniline polymerization. Different concentrations of oxidizing agent (APS, 0.25 and $0.50 \mathrm{M}$ ) and monomer (ANi, 0.50, 1.00 and $1.50 \mathrm{M}$ ), as well as different periods of polymerization $(30 \mathrm{~min} / 60 \mathrm{~min}$ in ANi followed by $3 \mathrm{hr} / 6 \mathrm{hr}$ in Ani and APS) were used. Statistical analysis was performed using a Kruskal-Wallis test followed by Dunn's test. Polymerization time and different monomer/oxidizing agent concentrations were compared between them and analyzed.

Table 2

Illustrative representation of synthetic and natural fabrics containing PPy or PANi.

\begin{tabular}{llll}
\hline Dyeing/polymerization time & Fabrics & PPy & PANi \\
\cline { 2 - 4 } & Oxidizing Agent (M) & 0.25 & 0.50 \\
\cline { 2 - 4 } & Monomer (M) & 0.50 & 0.50 \\
\hline $\begin{array}{c}\text { 30min Monomer/3hr Monomer and } \\
\text { Oxidizing Agent }\end{array}$ & Polyamide & \\
$\begin{array}{c}\text { 30min Monomer/3hr Monomer and } \\
\text { Oxidizing Agent } \\
\begin{array}{c}\text { 60min Monomer/6hr Monomer and } \\
\text { Oxidizing Agent }\end{array}\end{array}$ & $\begin{array}{l}\text { Plasma-treated } \\
\text { Polyamide }\end{array}$ & \\
\hline
\end{tabular}

Table 3

Macroscopic images of synthetic and natural fabrics containing PPy or PANi.

\begin{tabular}{|c|c|c|c|}
\hline \multirow[t]{3}{*}{ Dyeing/polymerization time } & Fabrics & PPy & PANi \\
\hline & $\begin{array}{l}\text { Oxidizing Agent } \\
\text { (M) }\end{array}$ & 0.25 & 0.50 \\
\hline & Monomer (M) & 0.50 & 0.50 \\
\hline $\begin{array}{l}\text { 30min Monomer/3hr Monomer and } \\
\text { Oxidizing Agent }\end{array}$ & Polyamide & & Fe \\
\hline $\begin{array}{l}\text { 30min Monomer/3hr Monomer and } \\
\text { Oxidizing Agent }\end{array}$ & $\begin{array}{l}\text { Plasma-treated } \\
\text { Polyamide }\end{array}$ & & (2) \\
\hline $\begin{array}{l}\text { 60min Monomer } / 6 \mathrm{hr} \text { Monomer and } \\
\text { Oxidizing Agent }\end{array}$ & Cotton & & \\
\hline
\end{tabular}

L929 cells were seeded on 96 well-plates at a cell density of $1 \times 10^{5}$ cells $/ \mathrm{mL}$ and left to adhere overnight. Following, the total and diluted extracts were added to the cells and incubated for $24 \mathrm{hr}$ and $72 \mathrm{~h}$ at $37^{\circ}$ and $5 \%$ of $\mathrm{CO}_{2}$. After these periods of incubation/culture, the metabolic activity of L929 cells was measured using the tetrazolium compound [3-(4,5-dimethylthiazol-2-yl)-5-(3-carboxymethoxyphenyl)2-(4-sulphophenyl)-2H-tetrazolium] (MTS colorimetric assay, Cell Titer 96 AQueous One Solution Cell Proliferation Assay, Promega, USA). Briefly, a mixture of serum-free cell culture medium and without phenol red and MTS reagent (CellTiter $96^{\circledR}$ AQueous One Solution Reagent) in a ratio of 5:1 was prepared and added to L929 cells for $3 \mathrm{~h}$ at $37^{\circ}$ and $5 \%$ of $\mathrm{CO}_{2}$, protected from light. Then, $100 \mu \mathrm{l}$ of MTS reagent was added to the wells of a 96-well plate and the absorbance was read at $490 \mathrm{~nm}$ using a microplate reader (Synergy HT, Bio-Tek, USA). Three independent experiments with triplicates were performed.

\subsection{Statistical analysis}

Statistical analysis was performed using the GraphPad software. Data were analyzed using the Shapiro-Wilk normality test and results did not present a normal distribution. Krustal-Wallis test with Dunn's multiple comparison post-test was used for statistical analysis. The significance level between groups were set for *p $<0.05$, **p $<0.01$ and $* * * \mathrm{p}<0.001$. Data were presented as mean \pm standard deviation (SD).

\section{Results}

\subsection{Electrical conductivity measurements}

In Figs. 1 and 2 it is possible to identify the influence of the concentration of the monomers (Py or ANi, M) and oxidizing agent (APS, $\mathrm{M}$ ) and dyeing time (pre-established time that fabrics were immersed in monomer solution and oxidizing agent until completely monomer polymerization), on the surface and volumetric conductivities of the synthetic and natural fabrics.

Non-dyed synthetic and natural fabrics presented conductivity in the order of $10^{-16}<\sigma>10^{-13}$ (Fig. S1). After dyeing, most of the synthetic and natural fabrics presented a semiconductor behavior in the order of $10^{-7}<\sigma>10^{-4}$ (Table 4). Although showing a similar conductivity profile, volumetric conductivity was lower than the surface conductivity (Table 5). This tendency was observed in all bleached and dyed fabrics (Fig. S1-3) PA fabrics presented a semiconductor behavior when a lower and intermediate concentration of Py $(0.50$ and $1.00 \mathrm{M})$ was used, independently of the dyeing time and oxidizing agent concentration (Fig. 1A). PPA (plasma-treated polyamide) fabrics presented a semiconductor behavior except for conditions using higher amount of APS $(0.5 \mathrm{M})$ and $1.00 \mathrm{M}$ of Py, for longer polymerization time, and for 
Table 4

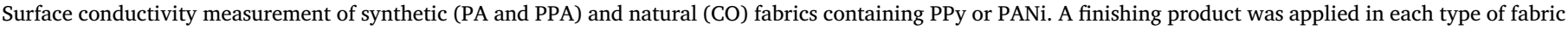
and both conductivities were measured and compared between them.

\begin{tabular}{|c|c|c|c|c|c|}
\hline \multicolumn{2}{|l|}{ Electrical Conductivity Measurement } & \multicolumn{2}{|l|}{ Fabrics containing PPy } & \multicolumn{2}{|c|}{ Fabrics containing PANi } \\
\hline Conditions & & Oxidizing Agent (M) & Monomer (M) & Oxidizing Agent (M) & Monomer (M) \\
\hline & & 0.25 & 0.50 & 0.50 & 0.50 \\
\hline \multirow[t]{2}{*}{ Dyeing time } & Finishing Product & $\mathrm{w} / \mathrm{o}$ & $\mathrm{w}$ & w/o & $\mathrm{w}$ \\
\hline & Fabrics & \multicolumn{4}{|c|}{ Surface Conductivity $\left(\sigma, S . \mathrm{cm}^{-1}\right)$} \\
\hline 30min Monomer/3hr Monomer and Oxidizing Agent & Polyamide & $1.27 \times 10^{-4}$ & $6.02 \times 10^{-5}$ & $1.06 \times 10^{-4}$ & $1.37 \times 10^{-5}$ \\
\hline 30min Monomer/3hr Monomer and Oxidizing Agent & Plasma-treated Polyamide & $8.80 \times 10^{-5}$ & $5.96 \times 10^{-5}$ & $1.11 \times 10^{-4}$ & $2.65 \times 10^{-5}$ \\
\hline 60min Monomer/6hr Monomer and Oxidizing Agent & Cotton & $6.77 \times 10^{-5}$ & $3.84 \times 10^{-5}$ & $2.58 \times 10^{-5}$ & $7.34 \times 10^{-6}$ \\
\hline
\end{tabular}

Table 5

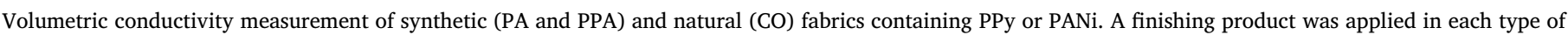
fabric and both conductivities were measured and compared between them.

\begin{tabular}{|c|c|c|c|c|c|}
\hline \multicolumn{2}{|l|}{ Electrical Conductivity Measurement } & \multicolumn{2}{|l|}{ Fabrics containing PPy } & \multicolumn{2}{|c|}{ Fabrics containing PANi } \\
\hline Conditions & & Oxidizing Agent (M) & Monomer (M) & Oxidizing Agent (M) & Monomer (M) \\
\hline & & 0.25 & 0.50 & 0.50 & 0.50 \\
\hline \multirow[t]{2}{*}{ Dyeing time } & Finishing Product & $\mathrm{w} / \mathrm{o}$ & $\mathrm{w}$ & w/o & $\mathrm{w}$ \\
\hline & Fabrics & \multicolumn{4}{|c|}{ Volumetric Conductivity $\left(\sigma, \mathrm{S}_{\mathrm{cm}}{ }^{-1}\right)$} \\
\hline 30min Monomer/3hr Monomer and Oxidizing Agent & Polyamide & $7.27 \times 10^{-6}$ & $6.69 \times 10^{-6}$ & $6.84 \times 10^{-6}$ & $5.69 \times 10^{-6}$ \\
\hline 30min Monomer/3hr Monomer and Oxidizing Agent & Plasma-treated Polyamide & $6.40 \times 10^{-6}$ & $6.90 \times 10^{-6}$ & $6.36 \times 10^{-6}$ & $7.36 \times 10^{-6}$ \\
\hline 60min Monomer/6hr Monomer and Oxidizing Agent & Cotton & $8.65 \times 10^{-6}$ & $6.36 \times 10^{-6}$ & $3.71 \times 10^{-6}$ & $5.49 \times 10^{-6}$ \\
\hline
\end{tabular}
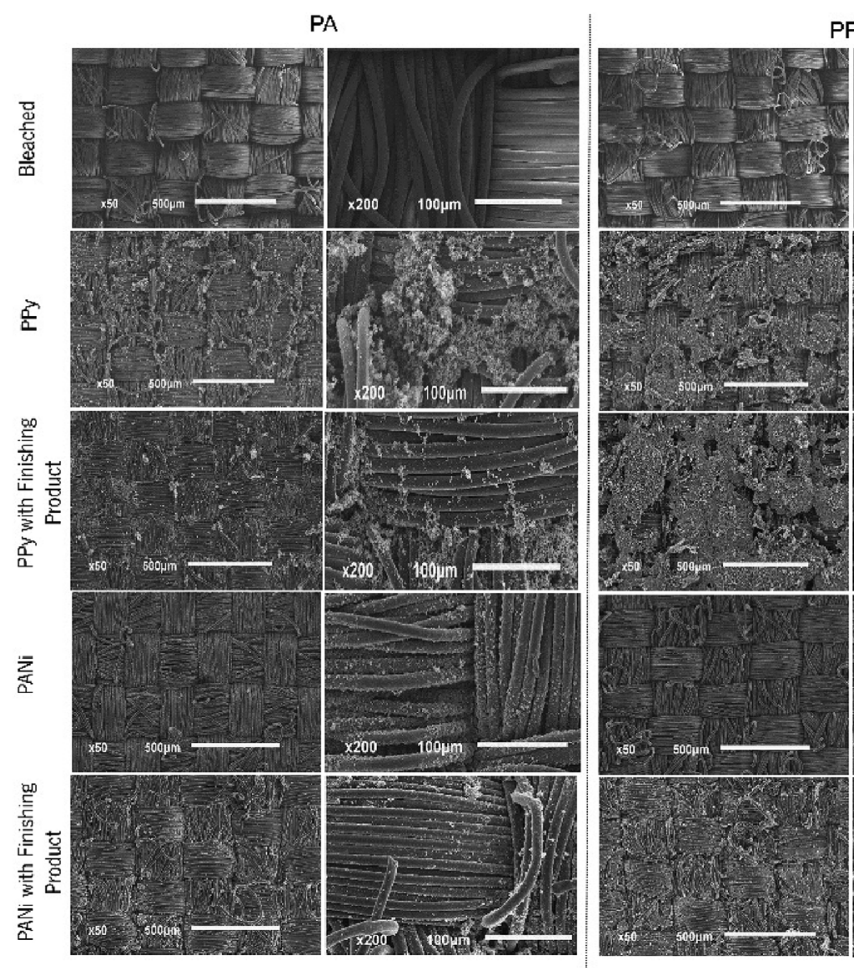

PPA

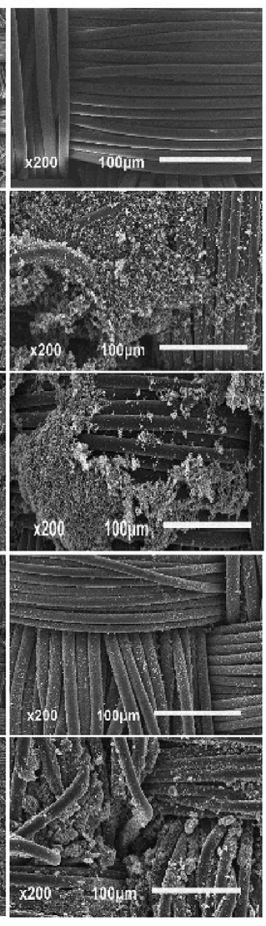

$\mathrm{CO}$

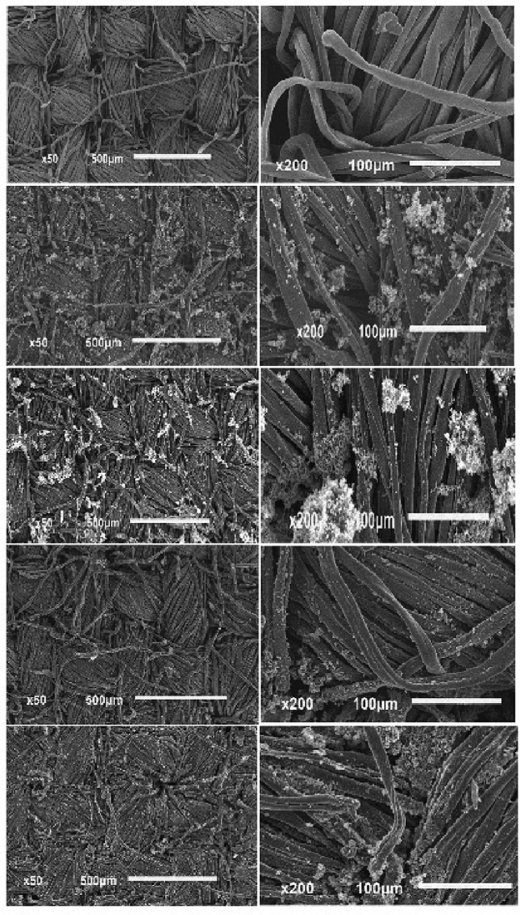

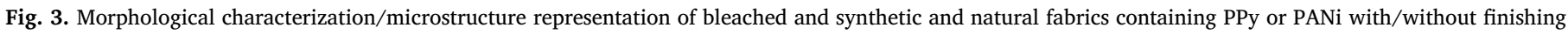
product.

$1.50 \mathrm{M}$ of Py independently of the polymerization time. PPA fabrics dyed with $0.50 \mathrm{M}$ of Py showed a significantly higher surface conductivity in relation to fabrics polymerized with $1.50 \mathrm{M}$ of Py and the same amount of APS ( $\sigma \approx 10^{-14}$ to $\left.\sigma \approx 10^{-4} ; * *, p<0,0076\right)$.
Natural fabrics (CO) (Fig. 1C) showed semiconductive properties, independently of the conditions used. Additionally, for the same Py concentration $(1.50 \mathrm{M})$ and lower dyeing time (30 min Py/3 h Py and APS), fabrics polymerized with $0.25 \mathrm{M}$ of APS showed a significantly 


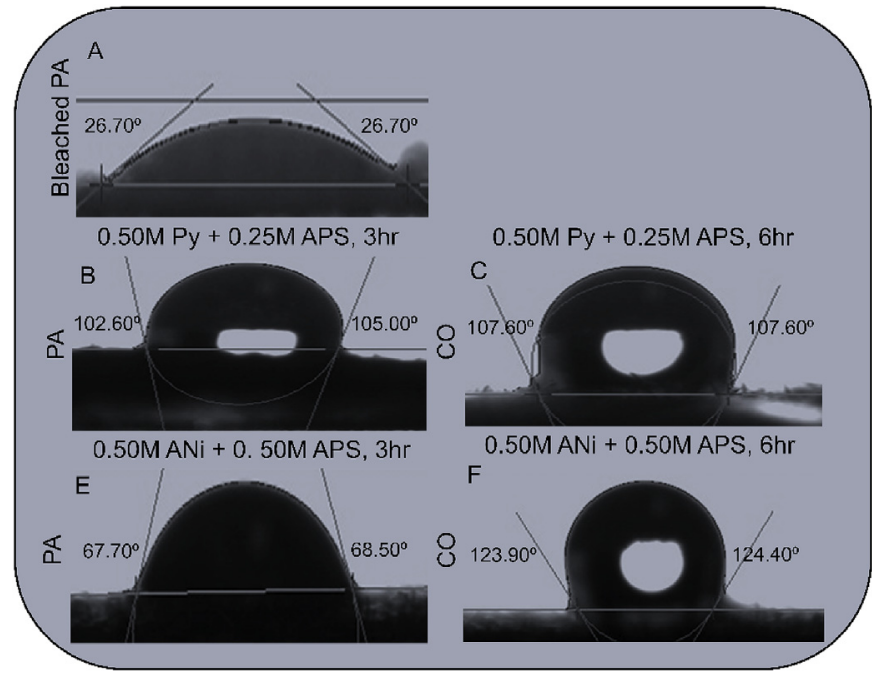

Fig. 4. Static contact angle of bleached PA (A), PA (B) and CO (C) fabrics dyed using $0.50 \mathrm{M}$ of Py and 0.25 M of APS and PA (E) and CO (F) fabrics dyed using $0.50 \mathrm{M}$ of $\mathrm{ANi}$ and $0.50 \mathrm{M}$ of APS. Data were presented in mean \pm standard deviaton. higher surface conductivity (*, $\mathrm{p}<0.0301$ ) in relation to fabrics dyed with $0.50 \mathrm{M}$ of APS.

All PA fabrics containing PANi (Fig. 2A) presented a semiconductor behavior except PA fabrics dyed with $1.50 \mathrm{M}$ of ANi, $0.25 \mathrm{M}$ of APS and longer polymerization time. In particular, fabrics dyed using $0.50 \mathrm{M}$ of ANi and $0.25 \mathrm{M}$ of APS and longer polymerization time, 60min ANi/6hr ANi and APS, showed significantly higher surface conductivity $\left(\sigma \approx 10^{-4}\right)$ in relation to PA fabrics dyed with $1.50 \mathrm{M}$ of $\mathrm{ANi}$ $\left(\sigma \approx 10^{-9}\right)(*, p<0.0281)$. Furthermore, the increase of ANi concentration $(0.50-1.50 \mathrm{M})$, seems to be the predominant factor on conductivity decrease. The same conductivity trend was observed in PPA and $\mathrm{CO}$ fabrics containing PANi, since all conditions showed semiconductive properties with exception of, but not significantly, both fabrics dyed using $1.50 \mathrm{M}$ of ANi and $0.25 \mathrm{M}$ of APS, in both polymerization times.

Based on these previous conductivity results (Figs. 1 and 2), the synthetic and natural fabrics that assembled the higher values of conductivity, as well as a better dimensional stability, uniformity and homogeneity of the dyeing (Tables S1-4) were selected for further analysis. In that sense, the best performing fabrics containing PPy required the lowest concentrations of Py $(0.50 \mathrm{M})$ and oxidizing agent $(0.25 \mathrm{M})$. Fabrics containing PANi required the lower concentration of ANi $(0.50 \mathrm{M})$ and the higher concentration of oxidizing agent $(0.50 \mathrm{M})$. Moreover, it is also important to highlight that synthetic fabrics containing PPy or PANi needed lower polymerization time when compared to the natural (CO) fabrics to achieve higher conductivity values. A
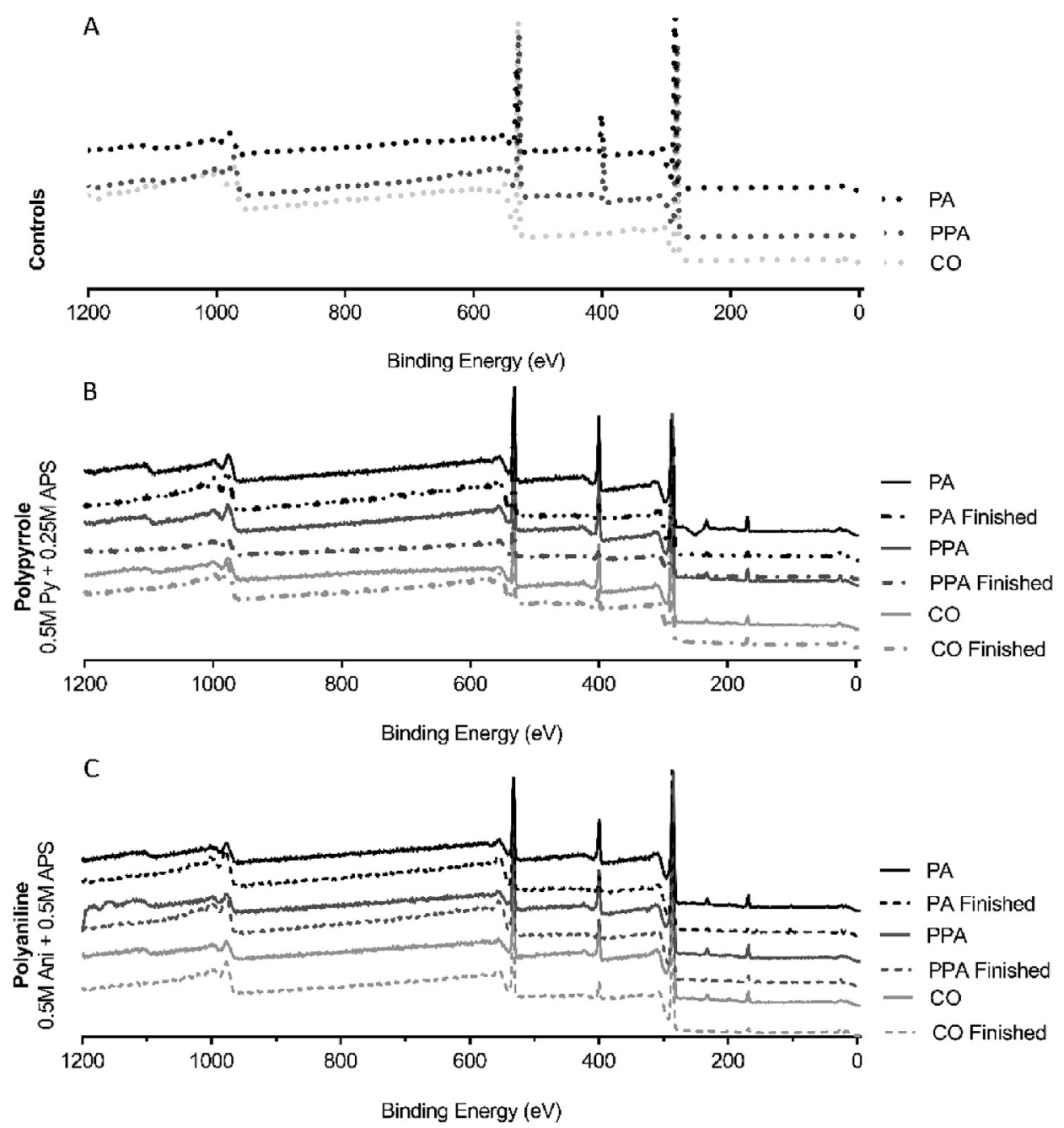

Fig. 5. XPS survey spectrum from bleached (A) and synthetic and natural fabrics containing PPy (B) or PANi (C). At each dyeing condition of synthetic and natural fabrics containing PPy or PANi was applied a finishing product. 
Table 6

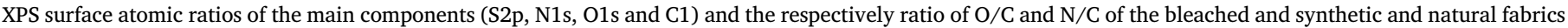
containing PPy or PANi. At each dyeing condition of synthetic and natural fabrics containing PPy or PANi was applied a finishing product.

\begin{tabular}{|c|c|c|c|c|c|c|c|}
\hline & & \multicolumn{4}{|c|}{ Atomic percentage } & \multicolumn{2}{|c|}{ Ratios } \\
\hline & & S 2p & $\mathrm{N} 1 \mathrm{~s}$ & O 1s & C $1 \mathrm{~s}$ & $\mathrm{O} / \mathrm{C}$ & $\mathrm{N} / \mathrm{C}$ \\
\hline \multirow[t]{3}{*}{ Control } & Polyamide (PA) & - & $9.59 \pm 0.22$ & $12.29 \pm 0.23$ & $78.11 \pm 0.31$ & 0.16 & 0.12 \\
\hline & Plasma-treated Polyamide (PPA) & - & $9.73 \pm 0.27$ & $19.55 \pm 0.17$ & $70.72 \pm 0.27$ & 0.28 & 0.14 \\
\hline & Cotton $(\mathrm{CO})$ & - & - & $31.39 \pm 0.17$ & $68.61 \pm 0.17$ & 0.46 & - \\
\hline \multirow[t]{6}{*}{ PPy } & PA - 0.5 M Py +0.25 M APS, $3 \mathrm{hr}$ & $1.59 \pm 0.03$ & $12.08 \pm 0.30$ & $13.73 \pm 0.23$ & $72.59 \pm 0.35$ & 0.19 & 0.17 \\
\hline & PA - 0.5 M Py + 0.25 M APS with Finishing Product, 3hr & $1.19 \pm 0.09$ & $3.01 \pm 0.28$ & $22.20 \pm 0.26$ & $73.60 \pm 0.37$ & 0.30 & 0.04 \\
\hline & $\mathrm{PPA}-0.5 \mathrm{M} \mathrm{Py}+0.25 \mathrm{M} \mathrm{APS}, 3 \mathrm{hr}$ & $1.58 \pm 0.03$ & $11.19 \pm 0.31$ & $16.26 \pm 0.27$ & $70.97 \pm 0.36$ & 0.23 & 0.16 \\
\hline & PPA - 0.5 M Py +0.25 M APS with Finishing Product, $3 \mathrm{hr}$ & $0.83 \pm 0.06$ & $4.02 \pm 0.28$ & $21.91 \pm 0.55$ & $73.24 \pm 0.60$ & 0.30 & 0.05 \\
\hline & $\mathrm{CO}-0.5 \mathrm{M} \mathrm{Py}+0.25 \mathrm{M}$ APS, $6 \mathrm{hr}$ & $1.12 \pm 0.03$ & $10.52 \pm 0.40$ & $12.93 \pm 0.29$ & $75.43 \pm 0.47$ & 0.17 & 0.14 \\
\hline & CO -0.5 M Py +0.25 M APS with Finishing Product, $6 \mathrm{hr}$ & $0.71 \pm 0.02$ & $4.76 \pm 0.17$ & $20.20 \pm 0.19$ & $74.33 \pm 0.23$ & 0.27 & 0.06 \\
\hline \multirow[t]{6}{*}{ PANi } & PA - 0.5 M Ani + 0.5 M APS, 3hr & $1.53 \pm 0.03$ & $9.00 \pm 0.28$ & $10.89 \pm 0.18$ & $78.59 \pm 0.31$ & 0.14 & 0.11 \\
\hline & PA - 0.5 M Ani + 0.5 M APS with Finishing Product, $3 \mathrm{hr}$ & $0.54 \pm 0.02$ & $3.02 \pm 0.13$ & $19.97 \pm 0.18$ & $76.47 \pm 0.22$ & 0.26 & 0.04 \\
\hline & PPA - 0.5 M Ani + 0.5 M APS, 3hr & $1.69 \pm 0.04$ & $6.97 \pm 0.26$ & $11.28 \pm 0.22$ & $80.05 \pm 0.34$ & 0.14 & 0.09 \\
\hline & PPA - 0.5 M Ani + 0.5 M APS with Finishing Product, 3hr & $0.65 \pm 0.02$ & $3.63 \pm 0.14$ & $19.80 \pm 0.17$ & $75.92 \pm 0.20$ & 0.26 & 0.05 \\
\hline & CO - 0.5 M Ani + 0.5 M APS, $6 \mathrm{hr}$ & $1.42 \pm 0.03$ & $9.02 \pm 0.24$ & $10.88 \pm 0.18$ & $78.68 \pm 0.28$ & 0.14 & 0.11 \\
\hline & CO -0.5 M Ani +0.5 M APS with Finishing Product, $6 \mathrm{hr}$ & $0.60 \pm 0.02$ & $3.58 \pm 0.17$ & $17.77 \pm 0.19$ & $78.06 \pm 0.23$ & 0.23 & 0.05 \\
\hline
\end{tabular}

Table 7

Color fastness testing in textiles: staining evaluation of adjacent fabrics that occurred with washing of a specimen at synthetic (PA and PPA) and natural (CO) fabrics containing PPy with/without finishing product.

\begin{tabular}{lllllllll}
\hline \multicolumn{2}{l}{ Staining evaluation - ISO A04 Index } & & & & & \\
\hline Fabrics containing PPy & & PA & & PPA & \multicolumn{2}{c}{ CO } \\
\hline \multirow{2}{*}{ Finishing Product } & & w/o & w & w/o & w & w/o & W \\
\hline \multirow{2}{*}{ Multifiber composition } & Wool & 4 & $4-5$ & 4 & 4 & $3-4$ & $3-4$ \\
& Polyamide & 4 & $3-4$ & 3 & 4 & $2-3$ & $3-4$ \\
& Cotton & 4 & 4 & 4 & 4 & $2-3$ & 3 \\
\hline
\end{tabular}

Table 8

Color fastness testing in textiles: staining evaluation of adjacent fabrics that occurred with washing of a specimen at synthetic (PA and PPA) and natural (CO) fabrics containing PANi with and without finishing product.

\begin{tabular}{llllllll}
\hline Fabrics containing PANi & PA & \multicolumn{3}{c}{ PPA } & \multicolumn{2}{c}{ CO } \\
\hline Finishing Product & & w/o & w & w/o & w & w/o & w \\
\hline \multicolumn{2}{l}{ Staining evaluation - ISO A04 Index } & & & & & & \\
Multifiber composition & Wool & $3-4$ & 4 & 3 & 4 & $3-4$ & $3-4$ \\
& Polyamide & $3-4$ & $3-4$ & 3 & 3 & 3 & 3 \\
& Cotton & 4 & $3-4$ & 4 & 4 & 4 & 3 \\
\hline
\end{tabular}

finishing product, BAYPRET ${ }^{\circledast}$ NANO-PU, composed of polyurethane was applied to the selected formulations of synthetic and natural fabrics containing PPy or PANi. The following experiments were performed using or not the finishing product and fabrics main properties were characterized.

\subsection{Macroscopic analysis and electrical conductivity measurements}

Macroscopic analysis reviled that fabrics containing PPy or PANi presented the characteristic color of each conductive polymer, black or green, respectively, as demonstrated on Table 2. Fabrics containing PPy or PANi showed homogeneous dyeing and stability (see Tables 2 and 3).

After the finishing product application, surface and volumetric conductivities were measured in the selected conditions and compared with the previous results (without finishing treatment). It was observed that the finishing process induced a decrease on final superficial conductivity, although not statistically significant, and no differences were observed regarding the volumetric conductivity (Figs. S2-3).

\subsection{Morphological analysis by scanning electron microscopy (SEM)}

Scanning electron microscopy (SEM) allowed the evaluation of microstructural alterations, dyeing uniformity and deposition of polymer precipitate between the bleached fabrics and fabrics containing PPy or PANi and between fabrics with and without coating with finishing product.

Synthetic and natural fabrics containing PPy or PANi demonstrated a uniform dyeing (Fig. 3). In both cases, the conductive polymer aggregated and was evidenced as a precipitate with a granular morphology on the top the synthetic and natural fibers. Moreover, both fabrics containing PPy showed higher deposition of polymer precipitate in comparison to fabrics containing PANi. Independently of the conductive polymer used, PA, PPA and CO fabrics treated with finishing product showed a gradually change in the surface morphology consisting of a significantly higher deposition of polymer precipitate.

\subsection{Contact angle measurements}

Contact angle measurements for the conditions involving PPA and CO fabrics are not shown due to their high hydrophilicity. PA fabrics presented a left and a right contact angle of $26.70^{\circ}$ (Fig. 4A). After Py polymerization $(0.50 \mathrm{M}$ Py $+0.25 \mathrm{M}$ APS), PA fabrics presented a left contact angle of $102.60^{\circ}$ and a right angle of $105.00^{\circ}$ (Fig. 4B), and CO fabrics showed a left and a right contact angle of $107.60^{\circ}$ (Fig. 4C), demonstrating an increase of hydrophobicity. Similarly, PA and CO containing PANi $(0.50 \mathrm{M}$ ANi $+0.50 \mathrm{M}$ APS $)$ showed a left contact angle of $67.70^{\circ}$ and $123.90^{\circ}$ and a right contact angle of $68.50^{\circ}$ and $124.40^{\circ}$, respectively (Fig. 4D-E). PPA fabrics containing PPy or PANi did not present any hydrophobicity even after monomers polymerization.

\subsection{X-ray photoelectron spectroscopy (XPS analysis)}

The presence of C1s, O1s, S2p and N1s was analyzed on all fabrics. The presence of the characteristic peaks of synthetic (PA and PPA) and natural (CO) fabrics was confirmed (Fig. 5A). These peaks were observed at 284.8 and $\approx 286 \mathrm{eV}$, respectively attributed to the $\mathrm{C}-\mathrm{C}$ and $\mathrm{C}-\mathrm{O}-\mathrm{C}$ groups of both synthetic and natural fabrics. Additionally, PA and PPA fabrics showed the presence of peaks at 288.5 (C 1s region spectrum), 400 and $\approx 533 \mathrm{ev}$ (O 1s region spectrum) which correspond, respectively, to the $0-\mathrm{C}=\mathrm{O}, \mathrm{C}-\mathrm{NH}_{2}$ and Organic $\mathrm{C}=\mathrm{O}$ groups of synthetic fabrics. In addition, the surface atomic ratios of the main components (S2p, N1s, O1s and $\mathrm{C} 1$ ) and the respectively ratio of $\mathrm{O} / \mathrm{C}$ and N/C of synthetic (PA and PPA) and natural (CO) fabrics was 

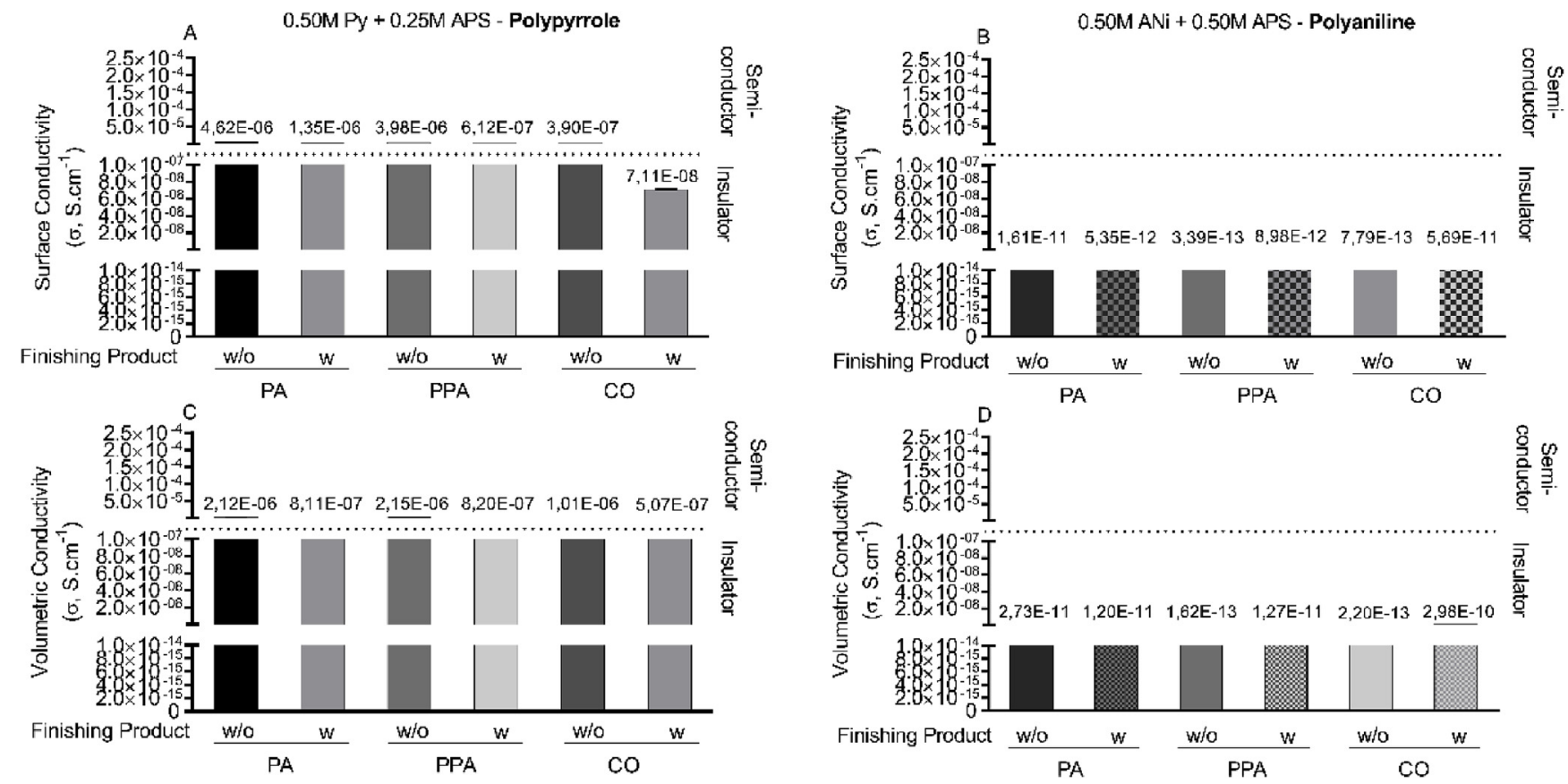

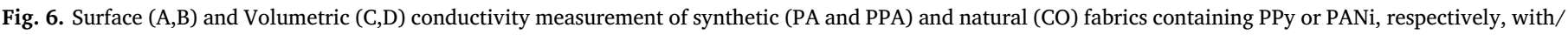
without finishing product after domestic and industrial washing.

Table 9

Color fastness to rubbing testing in textiles: staining evaluation of cotton adjacent fabric that occurred by rubbing in synthetic (PA and PPA) and natural (CO) fabrics containing PPy with/without finishing product.

\begin{tabular}{lllllll}
\hline Fabrics containing PPy & PA & \multicolumn{2}{c}{ PPA } & \multicolumn{2}{c}{ CO } \\
\hline Finishing Product & w/o & w & w/o & w & w/o & w \\
\hline $\begin{array}{l}\text { Staining evaluation - ISO A04 Index } \\
\text { Staining of cotton adjacent fabric }\end{array}$ & 1 & 1 & 1 & $1-2$ & 1 & 1 \\
\hline
\end{tabular}

\section{Table 10}

Color fastness to rubbing testing in textiles: staining evaluation of cotton adjacent fabric that occurred by rubbing in synthetic (PA and PPA) and natural (CO) fabrics containing PANi with/without finishing product.

\begin{tabular}{lcccccc}
\hline Fabrics containing PANi & PA & \multicolumn{2}{c}{ PPA } & \multicolumn{2}{c}{ CO } \\
\hline Finishing Product & w/o & w & w/o & w & w/o & w \\
\hline $\begin{array}{l}\text { Staining evaluation - ISO A04 Index } \\
\text { Staining of cotton adjacent fabric }\end{array}$ & $1-2$ & 1 & $1-2$ & 1 & 1 & 1 \\
\hline
\end{tabular}

demonstrated on Table 6.

It was possible to verify that the relative atomic concentration of Carbon decreased from PA (78.10\%) to PPA $(70.70 \%)$ fabrics, while the relative atomic concentration of Oxygen (from 12.28 to $19.53 \%$ ) and Nitrogen (from 9.57 to $9.70 \%$ ) increased. The CO fabrics had a relative atomic concentration of Carbon around $68.11 \%$ and Oxygen around $31.16 \%$.

Regarding to the PA fabrics containing PPy, additionally to the characteristics peaks of this type of fiber/fabric, it was observed the presence of sulfate ion/sulfate binding presenting a relative atomic concentration of Sulfate of $1.59 \%$. The same condition coated with finishing product presented an increase on the relative atomic concentrations of Carbon (from 72.59 to $76.49 \%$ ) and Oxygen (from 13.73 to $20.59 \%$ ) and a decrease on relative atomic concentration of Nitrogen (from 12.08 to $2.92 \%$ ).

Similarly, PPA fabrics containing PPy presented a relative atomic concentration of Sulfate of $1.58 \%$, Carbon of $70.9 \%$, Oxygen of $16.26 \%$ and Nitrogen of $11.19 \%$. Though, when the finishing product was applied to this condition, it was verified an increase on relative atomic concentrations of Carbon (73.24\%) and Oxygen (21.92\%) and a decrease on relative atomic concentration of Nitrogen (4.02\%) and Sulfate $(0.83 \%)$.

In respect to $\mathrm{CO}$ fabrics containing PPy, in addition to the characteristics peaks derived from the chemical structure of $\mathrm{CO}$, it was also verified the presence of the peaks corresponding to $\mathrm{C}-\mathrm{NH}_{2}$ groups and sulfate ion/binding. In addition, the CO fabrics containing PPy with finishing product, presented similar relative atomic concentrations of Carbon ( $\approx 74.88 \%$ ), an increase of Oxygen (from 12.93 to $20.20 \%$ ) and a decrease on the relative atomic concentration of Nitrogen (from 10.52 to $4.76 \%$ ) and Sulfate (from 1.12 to $0.71 \%$ ) comparing with CO fabrics containing PANi.

Concerning fabrics containing PANi, it was verified a similar trend as fabrics containing PPy. Related to the application of finishing product at PA fabrics containing PANi, it was observed that the finishing product application promoted a decrease on the relative atomic concentration of Sulfate (from 1.53 to $0.54 \%$ ), Carbon (from 78.59 to $76.47 \%$ ) and Nitrogen (from 9.00 to $3.02 \%$ ), while the relative atomic concentration of Oxygen increased from 10.89 to $19.97 \%$.

PPA fabrics containing PANi presented similar relative atomic concentrations of Sulfate, Carbon, Nitrogen and Oxygen when compared with PA fabrics containing PANi, showing the same trend.

At $\mathrm{CO}$ fabrics containing PANi, two additional peaks appeared at $\approx 400 \mathrm{eV}$ corresponding to $\mathrm{C}-\mathrm{NH}_{2}$ groups and another corresponding to Sulfate ion/binding. Furthermore, comparing the $\mathrm{CO}$ fabrics containing PANi using or not the finishing product, it was observed that the relative atomic concentration of Sulfate (from 1.42 to $0.60 \%$ ) and of Nitrogen (from 9.02 to $3.58 \%$ ) decreased while of Oxygen increased (from 10.88 to $17.77 \%$ ) being the relative atomic concentrations of Carbon $(\approx 78.00 \%)$ maintained.

\subsection{Color fastness testing in textiles (NP EN ISO 105)}

3.6.1. Color fastness of domestic and industrial washing - NP EN ISO 105:C06:1994. 

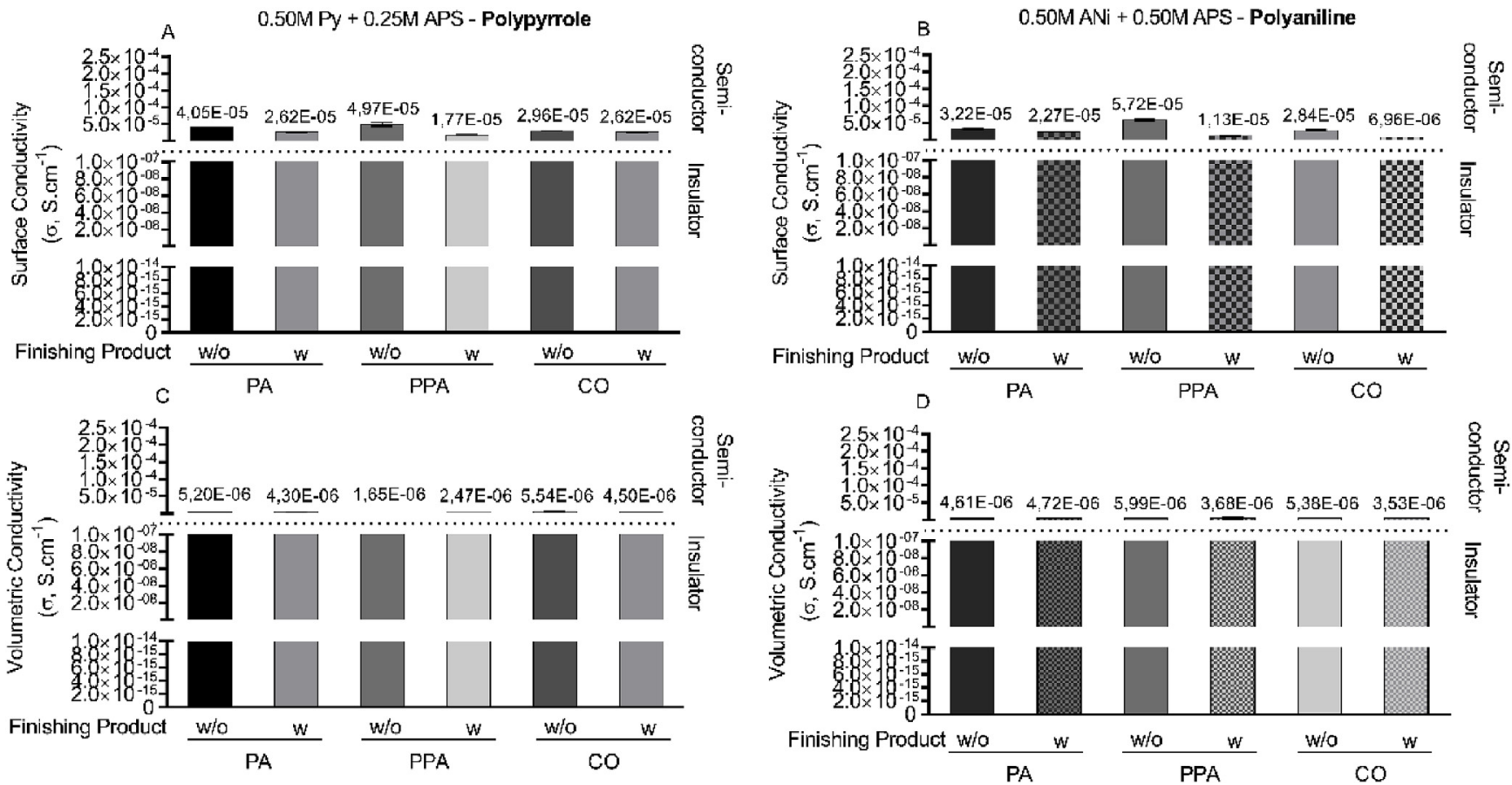

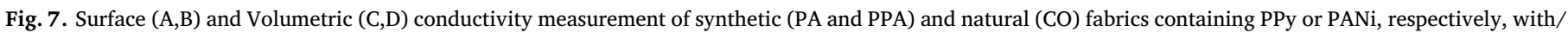
without finishing product after rubbing.

Color fastness of fabrics to multifiber fabric composed of diacetate, bleached cotton, polyamide, polyester, acrylic and wool was studied. The staining of adjacent fabrics was only quantified at textiles/fabrics visually affected (wool, polyamide and bleached cotton). Diacetate, polyester and acrylic fabrics were not stained by fabrics containing PPy or PANi. The application of finishing product maintained and even improved the color fastness of textiles with PPy and PANi, as demonstrated at Tables 7 and 8. PA and PPA fabrics achieved similar and higher values of color fastness when compared to $\mathrm{CO}$ fabrics as demonstrated by the relatively lower values.

\subsubsection{Electrical conductivity measurements after domestic and industrial washing}

Fabrics containing PPy maintained their semiconductive (surface and volumetric) properties after domestic and industrial washing (Fig. 6A,C). Exceptionally, CO fabrics treated with the finishing product changed their surface semiconductor properties to insulator; contrary, the volumetric conductivity remained semiconductor. Nevertheless, a slight but not statistically significant decrease was observed on surface and volumetric conductivities in all conditions tested. In opposition, fabrics containing PANi completely changed their semiconductor (surface and volumetric) behavior to insulator, as observed at Fig. 6B,D.

3.6.3. Color fastness to rubbing in textiles - NP EN ISO 105:X12:2003.

Color fastness of synthetic and natural fabrics containing PPY or PANi with/without finishing product to adjacent cotton fabric was studied and showed the lowest qualification/values of color fastness, as demonstrated at Tables 9 and 10.

\subsubsection{Electrical conductivity measurements after rubbing}

In spite of the lower values of color fastness to rubbing (Tables 9 and 10), surface and volumetric conductivities were not affected in all fabrics containing PPy or PANi. Independently of the polymer used (PPy and PANi) and the application of finishing product at synthetic and natural fabrics, their semiconductor properties were maintained (Fig. 7A-D).

\subsection{Cytotoxicity assay}

The cytotoxic effects of potential leachable from synthetic and natural fabrics bleached or containing PPy or PANi on L929 cells were evaluated along 3 days of culture. Cells cultured in growth media represent the negative control for cytotoxicity. Cells were metabolically active after culture for $24 \mathrm{hr}$ in contact with extracts from bleached synthetic and natural fabrics, independently of their percentage. After $72 \mathrm{hr}$ of culture bleached synthetic and natural fabrics showed signs of cytotoxicity when compared to the negative control. Cell metabolic activity significantly decreased to $50 \%$ after $72 \mathrm{hr}$ of culture with total $(100 \%)$ and diluted (70 and 50\%) extract from synthetic fabrics, and to $60 \%$ after $72 \mathrm{hr}$ of culture with total (100\%) extract from natural fabrics (Fig. 8A and B). On the other side, cell metabolic activity remained high $(100 \%)$ after $72 \mathrm{hr}$ of cell contact with extract from fabrics containing PPy or PANi (expect for CO fabrics containing PANi), when compared to bleached fabrics (Fig. 8). The application of the finishing product on PA fabrics indicated signs of cytotoxicity as significant differences were observed when these conditions were compared to the negative control. In fact, cells cultured for $72 \mathrm{hr}$ with total $(100 \%)$ and diluted $(70 \%)$ extract from PA fabrics containing PPy and finishing product showed a significant decrease to $70 \%$ on metabolic activity when compared to the same condition without finishing product. This effect was not observed with the extracts from natural fabrics containing PPy, not even with the highest concentration (100\%), as cell metabolic activity was similar to the control (Fig. 8B and C). In fact, cells cultured for $72 \mathrm{hr}$ with diluted (30\%) extract from CO fabrics and finishing product showed a significant increase on metabolic activity when comparing to the same condition at $24 \mathrm{hr}$ of culture (see Fig. 9).

Similarly to synthetic fabrics with PPy and in contrast to PA bleached fabrics, synthetic fabrics containing PANi showed high cell metabolic activity after $24 \mathrm{hr}$ and $72 \mathrm{hr}$ of cell culture in contact with the extracts, independently of the leachable percentage. Once again, the application of the finishing product on PA fabrics indicated signs of cytotoxicity. In the particular case of cells cultured with total (100\%) extract from PPA fabrics containing PANi and finishing product, at both time points, a significant decrease to $50 \%$ on metabolic activity was 

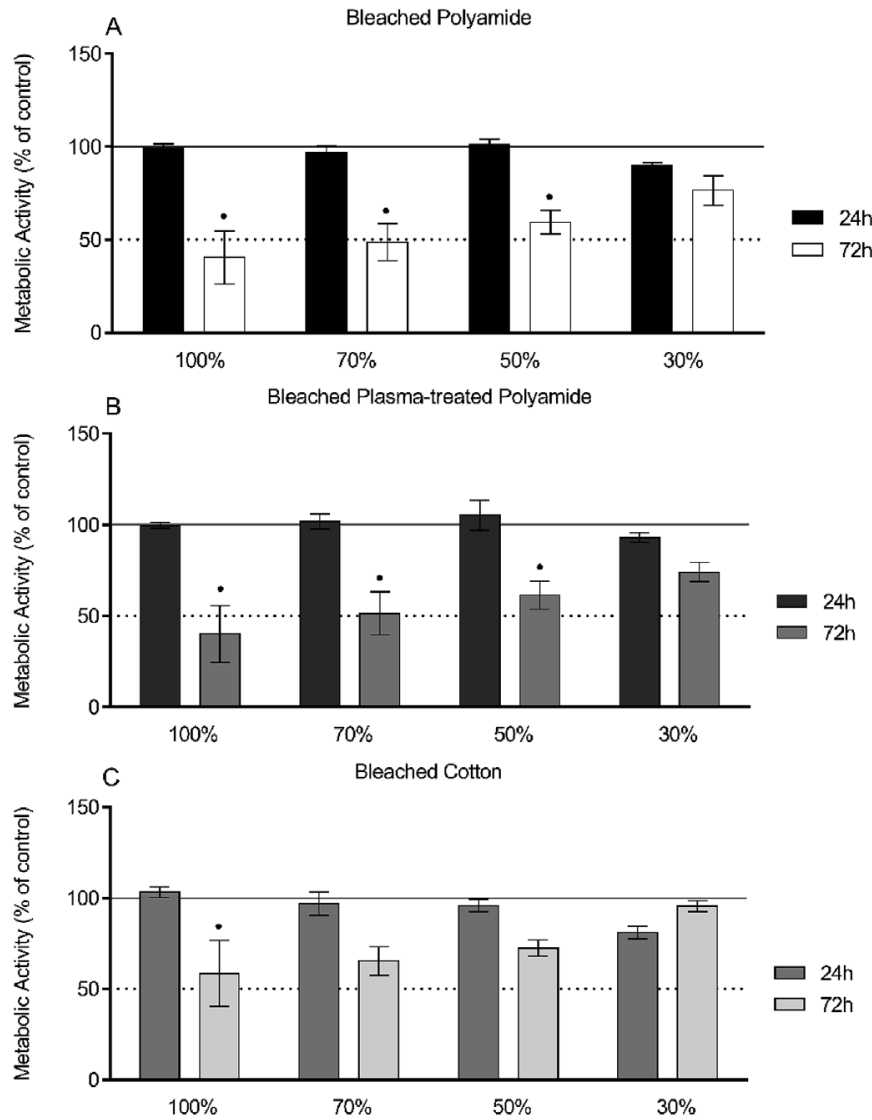

Fig. 8. Evaluation of the cytotoxic effects of potential leachables from bleached synthetic and natural fabrics, according to the ISO 10993-5:2009 - Biological evaluation of medical devices - Part 5: Tests for in vitro cytotoxicity. L929 cells were cultured for $24 \mathrm{hr}$ and $72 \mathrm{~h}$ at $37^{\circ}$ under a humidified atmosphere of $5 \% \mathrm{v} /$ $\mathrm{v} \mathrm{CO}_{2}$ in air in contact with total extracts (100\%) and diluted extracts $(70 \%$, $50 \%$ and $30 \%$ ) of bleached PA (A), PPA (B) and CO (C) fabrics in treatment medium. Metabolic activity of L929 cells was measured by MTS cytotoxicity assay and data were presented as percentage of control. Statistical analysis was performed using a Kruskal-Wallis test followed by Dunn's test and data was presented as mean \pm SD and symbols denote statistical differences $(\mathrm{p}<0.05)$ related to: $(\bullet)$ cells cultured for $24 \mathrm{hr}$, at the same concentration of extract, at the same finishing treatment.

observed when comparing to the same condition without finishing product. Moreover, this significant decrease was also observed between cells cultured for $72 \mathrm{hr}$ with total $(100 \%)$ extracts and diluted (50 and $30 \%)$ extracts with finishing product.

Signs of cytotoxicity were also observed when cells were cultured with leachable (100 and 70\%) of natural fabrics containing PANi as significant lower values of cell metabolic activity were observed in relation to the negative control of cytotoxicity. These values were lower than $\mathrm{CO}$ bleached fabrics. The cytotoxic effect of leachable was reduced when used at 50 and $30 \%$ (Fig. 10C). In that sense, cells were only metabolically active when cultured with the lower concentrations of diluted (50 and 30\%) extracts.

\section{Discussion}

Advances in biosensor technology has been revolutionizing healthcare through the daily monitoring of biological factors to treat more specifically pathological conditions. Wearable conductive based-textiles appear as excellent candidates to integrate these electronic devices as they not only protect, support and promote an intimate contact with the skin but also, accurately detect a change on electrochemical behavior when a biosensor is applied. In this sense, herein we describe the preparation and characterization of the first stage of wearable conductive based-textiles using natural (cotton) and synthetic (polyamide) fabrics prepared by in situ polymerization of polypyrrole or polyaniline.

The first goal of this work was to add electrical conductivity to the bleached synthetic and natural textiles. For this purpose, PPy and PANi were added to the textiles by in situ oxidative polymerization of the monomers on the top of the fabrics. It is well described in the literature that several polymerization parameters can affect the electrical conductivity of the final products [24]. Thus, in a first stage of this work, an experimental design was created (Table 1) aiming to optimize the polymerization process in order to achieve high values of electrical conductivity. With that purpose, different molar concentrations of monomers (Py and ANi) and APS and different dyeing/polymerization time were studied.

High values of surface and volumetric conductivities on fabrics were achieved using the lower concentrations $(0.50 \mathrm{M})$ of monomers (Py and ANi). However, the lower concentration of Py $(0.50 \mathrm{M})$ required the lower concentration of oxidizing agent $(0.25 \mathrm{M})$ to obtain higher conductivity values. In contrast, lower concentration of ANi $(0.50 \mathrm{M})$ required higher concentration of oxidizing agent $(0.50 \mathrm{M})$. Unexpectedly, conductive fabrics prepared with monomers at higher concentrations (Py or ANi, $1.50 \mathrm{M}$ ) showed the lowest conductivity values and the appearance and dimensional stability of the fabrics was affected since the dyeing was not homogeneous and the fabrics shrunk. It can be postulated that this effect occurs due to two different effects. If used at high concentrations, conducting polymers are deposited in textiles surface which then shrink. This may result in a lower diffusion of conductive polymers through the textiles which affects the final conductivity. Moreover, high concentrations of conductive polymer may cause a polymerization yield reduction. An over-oxidation converts a part of the PANI to quinone and PPy into a product would containing imine-like and amine-like nitrogens [25]. In addition, optimal polymerization time was dependent of the fabric used since synthetic fabrics containing PPy or PANi needed lower polymerization time to achieve higher conductivity values when compared to the natural (CO) fabrics. It happens due to the chemical structure of the synthetic and natural fabrics. In contrast to bleached $\mathrm{CO}$, synthetic fabrics present nitrogen groups that lead to an increased affinity between the conductive polymers and the fibers. This, results in a faster polymerization and increased conductivity values for synthetic fabrics containing PPy or PANi.

Volumetric conductivity was determined to verify if the monomer and its polymerization was effective inside the fabrics. The volumetric conductivity was similar but relatively lower when comparing to the surface conductivity (Figs. S2 and 3). This result can be explained by the deposition of conductive polymer aggregates on the surface of synthetic and natural fabrics, limiting the diffusion of monomers. Nevertheless, the integration of conducting polymers on synthetic and natural fabrics promoted a change in their conductive behavior, changing from the insulator form to semiconductor. The partial oxidation of these conducting polymers generates the appropriate charged carriers that are responsible for the semiconductor properties of the fabrics [26].

After selecting the best polymerization conditions, a finishing product was applied in both types of fabrics, resulting in a slight decrease of conductivity values. This treatment was applied with the aim to better preserve their characteristics. These fabrics were then characterized in terms of morphology, hydrophobicity, chemical composition, color fastness of domestic and industrial washing and to rubbing and cytotoxicity. Regarding the microstructural analysis of synthetic and natural fabrics, it was notorious the presence of higher amount of PPy precipitates and aggregates on the top of the fibers when comparing with fabrics containing PANi. The same occurred in both polymers after applying the finishing product. The in situ polymerization of conducting polymers in textile substrates comprises the deposition of polymer and precipitation of its homopolymer in the reaction bath 


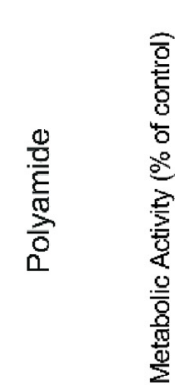

$$
\text { A }
$$

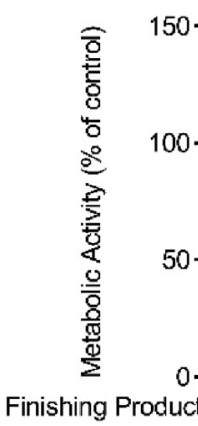

PA: 0.50M Py+0.25M APS

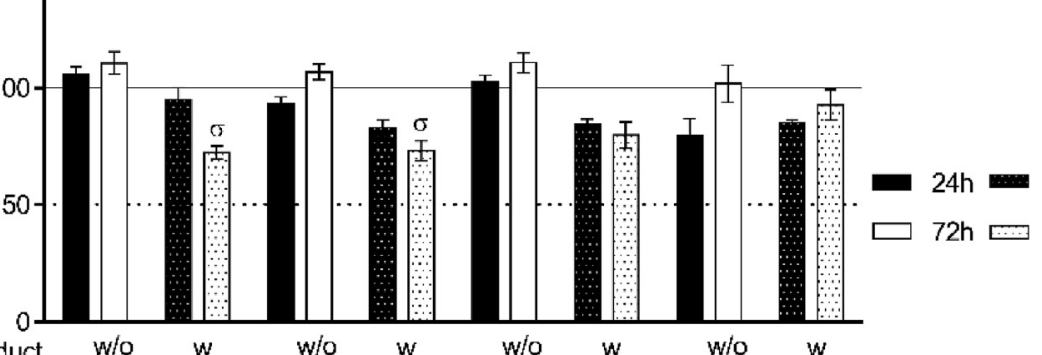

B

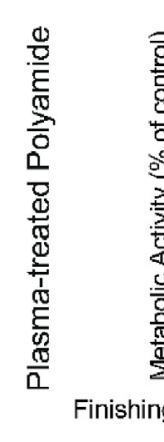

$100 \%$

w/o

w/o $w$ w/o $w$

PPA: 0.50M Py + 0.25M APS

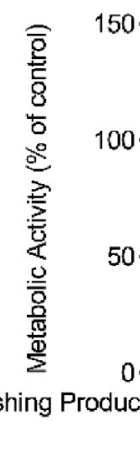

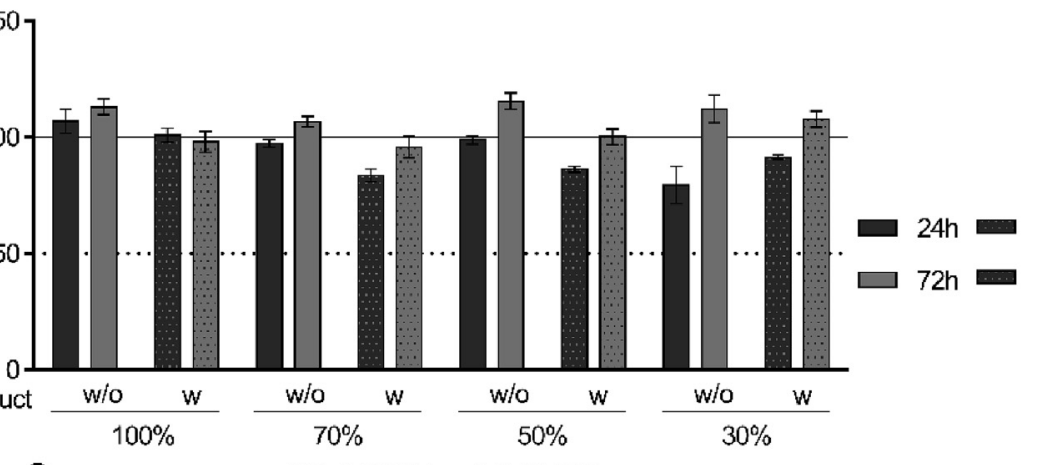

C

CO: $0.50 \mathrm{M}$ Py $+0.25 \mathrm{M}$ APS

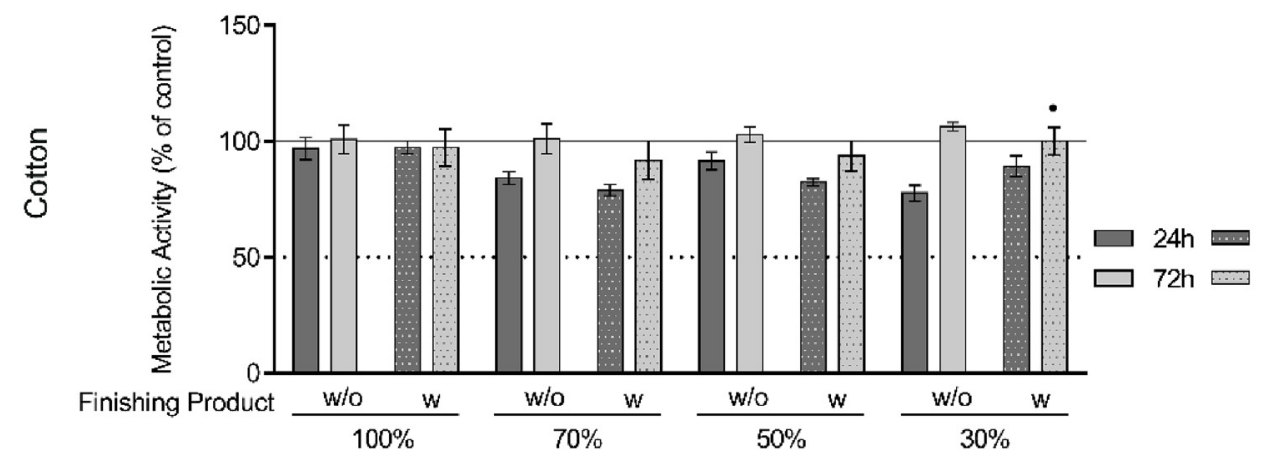

Fig. 9. Evaluation of the cytotoxic effects of potential leachables from synthetic and natural fabrics containing PPy, according to the ISO 10993-5:2009 - Biological evaluation of medical devices - Part 5: Tests for in vitro cytotoxicity. L929 cells were cultured for $24 \mathrm{hr}$ and $72 \mathrm{~h}$ at $37^{\circ}$ under a humidified atmosphere of $5 \% \mathrm{v} / \mathrm{v} \mathrm{CO}_{2}$ in air in contact with total extracts $(100 \%)$ and diluted extracts $(70 \%, 50 \%$ and $30 \%)$ of PA (A), PPA (B) and CO (C) fabrics containing PPy in treatment medium. Metabolic activity of L929 cells was measured by MTS cytotoxicity assay and data were presented as percentage of control. Statistical analysis was performed using a Kruskal-Wallis test followed by Dunn's test and data was presented as mean \pm SD and symbols denote statistical differences $(\mathrm{p}<0.05)$ related to: $(\sigma)$ cells cultured without finishing product, at the same concentration of extract, at the same time-point; $(\bullet)$ cells cultured for $24 \mathrm{hr}$, at the same concentration of extract, at the same finishing treatment.
[27]. The formation of a uniform layer of PPy or PANi particles involving the fibers and into the interstices was also reported in other studies, showing the effectiveness of the polymerization process [28]. It is also reported that the polymer precipitate and aggregates increased proportionally with the increasing of monomer and polymerization time, leading to the compaction of the polymer [29,30]. In this sense, parameters, such as fiber-polymer interaction, functional groups in the fiber, number of fibers in the equivalent weight of fabric, shape of the fiber, twist in the fiber, and fabric structure can influence the redistribution of contact points [28]. Considering the fiber-polymer interaction, fabrics containing PPy presented more polymer precipitate and aggregates on the top of the fibers when comparing to the fabrics containing PANi.

The Plasma treatments were performed on PA fabrics aiming to increase their hydrophilicity. The obtained results have shown that the microstructure of PA was not affected by plasma treatment. Furthermore, comparing PA and PPA fabrics, the increase of Oxygen groups provided by plasma-treatment promoted a better adhesiveness between the fibers and the conducting polymers/dyeing, as observed by their homogeneity. Clearly, the decrease of the relative atomic concentration of Carbon is accomplished by the increase of Oxygen and Nitrogen content due to the creation of reactive groups and radicals on the surface of the fabric allowing the formation of dipolar interactions, van der Waals forces or hydrogen bonds between the fabric and the coating/dyeing [31,32]. In addition, as reported in the literature, plasma treatment modifies chemically and physically polyamide fabrics, increasing the content of hydrophilic functional groups on the fiber surface as dosage applied is increased [23].This treatment did not influence the conductive behavior of the fabrics. Relatively to the synthetic and natural fabrics containing or not PPy or PANi, as expected, the relative atomic concentration of Oxygen was higher at $\mathrm{CO}$ fabrics when compared to PA/PPA fabrics. This is a result of the chemical structure of $\mathrm{CO}$ that contains higher amount of Oxygen. Moreover, the modification with PPy/PANi and APS promoted an increase of Sulphur concentration. Concerning the application of the finishing product at PA fabrics containing PPy, an increase of Carbon and Oxygen and a decrease on relative atomic concentration of Nitrogen and Sulphur was observed. The reduction of Sulphur and Nitrogen at the surface can be explained by ability of the finishing product to act as a thin surface coating. The finishing product, BAYPRET NANO PU, is composed of polyurethane which is known by their elasticity and has been widely used in the textile industry including the production of elastic yarns and fabrics (elastomer), as an adhesive and coating agent [33,34]. Overall, the rearrangement of the $\mathrm{O} / \mathrm{C}$ and $\mathrm{N} / \mathrm{C}$ ratios (Table 6) on fabrics 
A

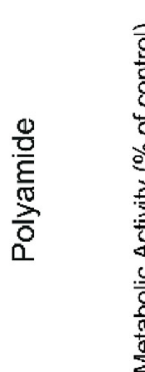

Finishing Product
PA: $0.50 \mathrm{M} \mathrm{ANi}+0.50 \mathrm{M}$ APS

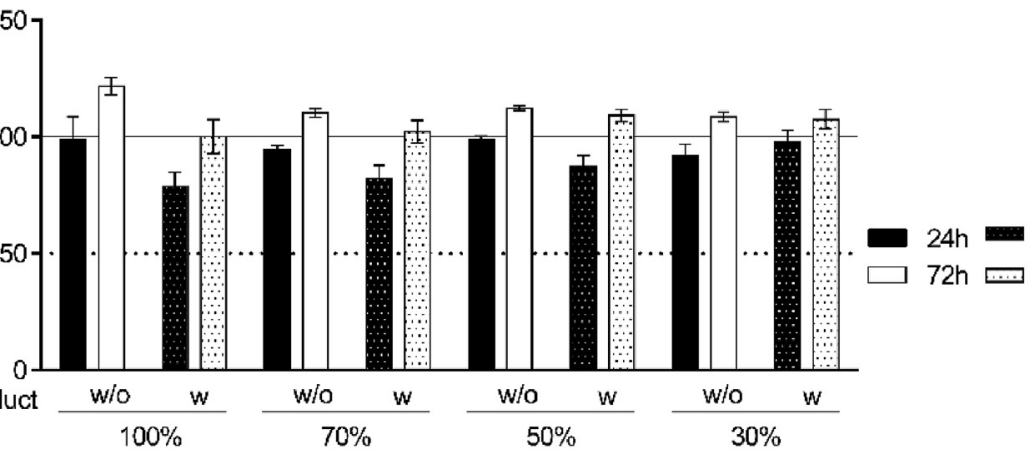

B

PPA: $0.50 \mathrm{M} \mathrm{ANi}+0.50 \mathrm{M}$ APS

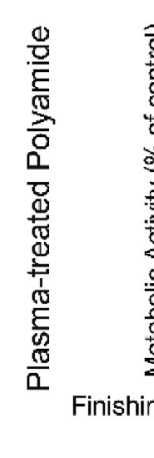

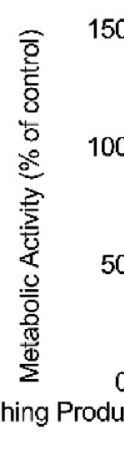

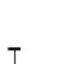


reported a dry-wash test using tetrachloroethylene, according to the standard test methods 132-2004 [35]. As an example, the surface resistance of the developed conductive textile through covalently grafting polyaniline (APGC-g-PANI) onto cotton showed to be stable even after 40 dry-wash cycles. It was not observed an obvious destruction of the PANi layer on the fiber after the dry-wash, indicating that the conductive network of the APGC-g-PANI fabric was able to withstand the washing process [35]. In a different study, Patil et al. tested the durability to washing through the color fastness to washing and to dry cleaning tests and reported a higher durability on dry samples than wet samples. The loss of conductivity happened due to the washing away of the dopant ions coupled with the attack of alkaline water on the polymeric chain [38].

The use of conductive polymers (PPy or PANi) on synthetic and natural fabrics enhanced the metabolic activity of L929 cells, surpassing the cytotoxicity observed for bleached synthetic and natural fabrics. All fabrics (synthetic or natural) contain impurities (natural or added during the manufacturing process) that are commonly removed prior dyeing in order to: i) increase the hydrophilicity of the fibers, ii) increase the absorption capacity of aqueous solutions of dyes and other chemicals, iii) provide a proper degree of whiteness, especially in the case of white and light colors and, iv) increase color yield. This process (bleaching) is generally carried out using alkaline baths and chemicals products, as hypochlorite and persalts. Therefore, we may postulate that the cytotoxicity of bleached synthetic and natural fabrics detected by indirect contact might result from the alkaline leachable released from bleached textiles. This was not detected in the fabrics containing PPy or PANi as these were subjected to an in situ polymerization process that not only involves several washing steps that might remove the cytotoxic leachable, but also modifies the final properties and structure of the fabrics, reducing the cytotoxicity. Additionally, the efficiency of plasma-treatment at PA fabrics containing PPy was also observed on cell behavior, as a higher and homogeneously metabolic activity was evidenced when comparing to the PA containing PPy.

All fabrics containing PPy and synthetic fabrics containing PANi did not show cytotoxicity, evidencing their suitability as substrates to be used in biomedical applications. However, leachables/extracts (specially, 100 and $70 \%$ ) from natural fabrics containing PANi promoted cytotoxicity. This was also evidenced in a previous work developed by Nela Marákováa et al., 2017 that reported that PANi-coated cotton was significantly more cytotoxic than PPy coated-cotton [39].

\section{Conclusion}

Wearable conductive based-textiles using synthetic and natural fabrics with semiconductive properties were herein developed by combining PA/CO with PPy/PANi. With our study, we stablished the optimal conditions to obtain synthetic and natural conductive fabrics by changing their conductive behavior from insulator to semiconductor. Additionally, we were able to develop electroactive textiles platforms with different structures, maintaining their integrity (as flexibility and durability) to open the range of applicability on biomedical field. L929 cells do not show nefarious effects in indirect contact with the conductive textiles, a result not achieved so far. Moreover, with the developed textiles it is possible to add some bio-functionality without impairing comfort. In addition, the conductive textiles can act as an interactive supporting textile platform to be applied in direct contact with the skin, keeping their flexibility and accurate sensibility to quantify/measure the stimuli (signals) studied/promoted.

Envisioning a final application on biomedical field, a biosensor/ electronic device can be applied within or on top of the conductive textiles and through electrical conductivity change measurement, the signal can be detected for a long period of time.

\section{Acknowledgements}

The authors thank the funds provided by FEDER funds through Operational Programme for Competitiveness Factors - COMPETE and National Funds through FCT - Foundation for Science and Technology within the scope of the projects POCI-01-0145-FEDER-007136 and UID/CTM/00264 and the grant POCI-01-0145-FEDER-007038UMINHO/BPD/44/2016 (LPS), and by the project FROnTHERA (NORTE-01-0145-FEDER-000023), supported by Norte Portugal Regional Operational Programme (NORTE 2020), under the PORTUGAL 2020 Partnership Agreement, through the European Regional Development Fund (ERDF).

\section{Appendix A. Supplementary data}

Supplementary data to this article can be found online at https:// doi.org/10.1016/j.orgel.2019.105401.

\section{References}

[1] G. Cappon, G. Acciaroli, M. Vettoretti, A. Facchinetti, G. Sparacino, Wearable continuous glucose monitoring sensors: a revolution in Diabetes treatment, Electronics 6 (3) (2017).

[2] L. Piwek, D.A. Ellis, S. Andrews, A. Joinson, The rise of consumer health wearables: promises and barriers, PLoS Med. 13 (2) (2016) e1001953.

[3] Wearable wireless sensors for chronic respiratory disease monitoring, in: J.P. Dieffenderfer, H. Goodell, B. Bent, E. Beppler, R. Jayakumar, M. Yokus, et al. (Eds.), IEEE 12th International Conference on Wearable and Implantable Body Sensor Networks (BSN); 2015 9-12 June 2015, 2015.

[4] A.J. Bandodkar, J. Wang, Non-invasive wearable electrochemical sensors: a review, Trends Biotechnol. 32 (7) (2014) 363-371.

[5] H.Y.Y. Nyein, W. Gao, Z. Shahpar, S. Emaminejad, S. Challa, K. Chen, et al., A wearable electrochemical platform for noninvasive simultaneous monitoring of $\mathrm{Ca} 2+$ and pH, ACS Nano 10 (7) (2016) 7216-7224.

[6] B.W. An, J.H. Shin, S.-Y. Kim, J. Kim, S. Ji, J. Park, et al., Smart sensor systems for wearable electronic devices, Polymers 9 (8) (2017).

[7] S. Nakata, T. Arie, S. Akita, K. Takei, Wearable, flexible, and multifunctional healthcare device with an ISFET chemical sensor for simultaneous sweat $\mathrm{pH}$ and skin temperature monitoring, ACS Sens. 2 (3) (2017) 443-448.

[8] Y. Liu, M. Pharr, G.A. Salvatore, Lab-on-Skin: a review of flexible and stretchable electronics for wearable health monitoring, ACS Nano 11 (10) (2017) 9614-9635.

[9] Yeo JC. Kenry, C.T. Lim, Emerging flexible and wearable physical sensing platforms for healthcare and biomedical applications, Microsyst. Nanoeng. 2 (2016) 16043.

[10] J. Bujes-Garrido, M.J. Arcos-Martínez, Development of a wearable electrochemical sensor for voltammetric determination of chloride ions, Sens. Actuators B Chem. 240 (2017) 224-228.

[11] R.S. Malon, K.Y. Chua, D.H. Wicaksono, E.P. Corcoles, Cotton fabric-based electrochemical device for lactate measurement in saliva, Analyst 139 (12) (2014) 3009-3016.

[12] G. Matzeu, L. Florea, D. Diamond, Advances in wearable chemical sensor design for monitoring biological fluids, Sens. Actuators B Chem. 211 (Supplement C) (2015) 403-418.

[13] A. Abdal-Hay, K. Abdelrazek Khalil, F.F. Al-Jassir, A.M. Gamal-Eldeen, Biocompatibility properties of polyamide 6/PCL blends composite textile scaffold using EA.hy926 human endothelial cells, Biomed. Mater. 12 (3) (2017) 035002.

[14] B. Pejic, A.M. Baralic, Z. Kojic, P. Skundric, M. Kostic, Oxidized cotton as a substrate for the preparation of hormone-active fibers-characterization, efficiency and biocompatibility, Fibers Polym. 16 (5) (2015) 997-1004.

[15] C.-S. Wu, Mechanical properties, biocompatibility, and biodegradation of crosslinked cellulose acetate-reinforced polyester composites, Carbohydr. Polym. 105 (Supplement C) (2014) 41-48.

[16] Lina MCaABF, Smart fabric sensors and e-textile technologies: a review, Smart Mater. Struct. 23 (5) (2014) 053001.

[17] T.K. Das, S. Prusty, Review on conducting polymers and their applications, Polym. Plast. Technol. Eng. 51 (14) (2012) 1487-1500.

[18] Y. Zhao, Y. Li, W. Kang, Y. He, W. Liu, H. Liu, et al., A novel flexible sensor for respiratory monitoring based on in situ polymerization of polypyrrole and polyurethane coating, RSC Adv. 7 (78) (2017) 49576-49585.

[19] Y. Huang, L. Gao, Y. Zhao, X. Guo, C. Liu, P. Liu, Highly flexible fabric strain sensor based on graphene nanoplatelet-polyaniline nanocomposites for human gesture recognition, J. Appl. Polym. Sci. 134 (39) (2017) 45340.

[20] F.V. Berti, P. Srisuk, L.P. da Silva, A.P. Marques, R.L. Reis, V.M. Correlo, * synthesis and characterization of electroactive gellan gum spongy-like hydrogels for skeletal muscle tissue engineering applications, Tissue Eng. A 23 (17-18) (2017) 968-979.

[21] Srisuk P, Berti F, Correlo V, Reis RL. Electroactive Spongy-like Hydrogels Based on Gellan Gum and Polyaniline for Tissue Engineering Applications. TERM STEM 2014 - Nano Tools for Diagnosis and Regeneration of Tissue; Porto2014.

[22] N. Carneiro, A.P. Souto, E. Prinz, F. Forster, inventorsContinuous and semi-continuous treatment of textile materials integrating CORONA discharge, Portugal (2004). 
[23] A.P. Souto, F.R. Oliveira, M. Fernandes, N. Carneiro, Influence of DBD plasma modification in the dyeing process of polyamide, J. Text. Eng. 19 (2012).

[24] A. Kaynak, R. Beltran, Effect of synthesis parameters on the electrical conductivity of polypyrrole-coated poly(ethylene terephthalate) fabrics, Polym. Int. 52 (6) (2003) 1021-1026.

[25] V. Blinova N, J. Stejskal, M. Trchová, J. Prokeš, M. Omastová, Polyaniline and polypyrrole: a comparative study of the preparation, Eur. Polym. J. 43 (2007) 2331-2341.

[26] M. Teli, S. Dash, P. Desai, Polyaniline based conductive textiles, J. Inst. Eng.: Ser. E. 95 (2) (2014) 75-79.

[27] A.J. Patil, S.C. Deogaonkar, A novel method of in situ chemical polymerization of polyaniline for synthesis of electrically conductive cotton fabrics, Text. Res. J. 82 (15) (2012) 1517-1530.

[28] S. Maity, A. Chatterjee, Textile/Polypyrrole Composites for Sensory Applications, (2015), p. 6.

[29] A. Varesano, L. Dall'Acqua, C. Tonin, A study on the electrical conductivity decay of polypyrrole coated wool textiles, Polym. Degrad. Stab. 89 (1) (2005) 125-132.

[30] V. Tunáková, J. Grégr, M. Tunák, G. Dohnal, Functional polyester fabric/polypyrrole polymer composites for electromagnetic shielding: optimization of process parameters, J. Ind. Text. (2016) 1528083716667262.

[31] J. Molina, M.F. Esteves, J. Fernández, J. Bonastre, F. Cases, Polyaniline coated conducting fabrics. Chemical and electrochemical characterization, Eur. Polym. J. 47 (10) (2011) 2003-2015.
[32] J. Molina, F.R. Oliveira, A.P. Souto, M.F. Esteves, J. Bonastre, F. Cases, Enhanced adhesion of polypyrrole/PW12O 403 - hybrid coatings on polyester fabrics, $\mathrm{J}$. Appl. Polym. Sci. 129 (1) (2013) 422-433.

[33] Z.-R. Li, W.-C. Jiang, L.-J. Wang, W.-D. Meng, F.-L. Qing, Synthesis and application of novel aqueous anionic polyurethane as a durable press finishing agent of cotton fabrics, Text. Res. J. 77 (4) (2007) 227-232.

[34] C. Wang, H. Meng, X. Wang, C. Zhao, J. Peng, Y. Wang, Differentiation of bone marrow mesenchymal stem cells in osteoblasts and adipocytes and its role in treatment of osteoporosis, Med. Sci. Monit. 22 (2016) 226-233.

[35] B. Wu, B. Zhang, J. Wu, Z. Wang, H. Ma, M. Yu, et al., Electrical switchability and dry-wash durability of conductive textiles, Sci. Rep. 5 (2015) 11255.

[36] R. Neelakandan, M. Madhusoothanan, Electrical resistivity studies on polyaniline coated polyester fabrics, J. Eng. Fiber Fabr. 3 (2009).

[37] N. Onar Çamlıbel, A. Akşit, F. Ebeoglugil, I. Birlik, E. Celik, I. Ozdemir, Structural, Electrical, and Electromagnetic Properties of Cotton Fabrics Coated with Polyaniline and Polypyrrole2009, (2003), p. 10.

[38] Patil A, K. Pandey A. A Novel Approach for in Situ Polymerization of Polypyrrole on Cotton Substrates2012. 107-113 p.

[39] N. Maráková, P. Humpolíček, V. Kašpárková, Z. Capáková, L. Martinková, P. Bober, et al., Antimicrobial activity and cytotoxicity of cotton fabric coated with conducting polymers, polyaniline or polypyrrole, and with deposited silver nanoparticles, Appl. Surf. Sci. 396 (Supplement C) (2017) 169-176. 